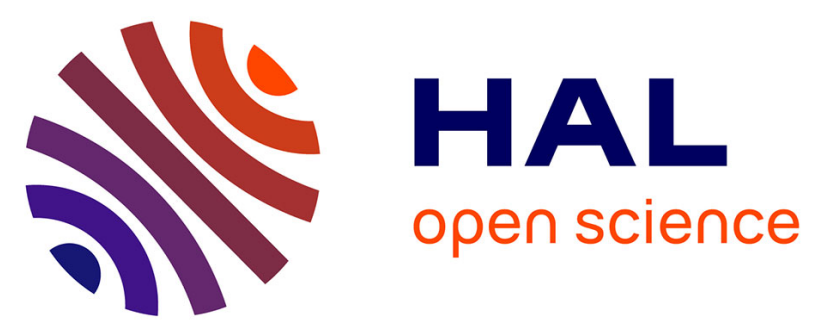

\title{
Theory, simulation, and observation of discrete eigenmodes associated with lower hybrid solitary structures
}

P.W. Schuck, C.E Seyler, Jean-Louis Pinçon, J.W. Bonnell, P.M. Kintner

\section{- To cite this version:}

P.W. Schuck, C.E Seyler, Jean-Louis Pinçon, J.W. Bonnell, P.M. Kintner. Theory, simulation, and observation of discrete eigenmodes associated with lower hybrid solitary structures. Journal of Geophysical Research Space Physics, 1998, 103 (A4), pp.6935-6953. 10.1029/97JA02922 . insu-03192014

\section{HAL Id: insu-03192014 \\ https://hal-insu.archives-ouvertes.fr/insu-03192014}

Submitted on 7 Apr 2021

HAL is a multi-disciplinary open access archive for the deposit and dissemination of scientific research documents, whether they are published or not. The documents may come from teaching and research institutions in France or abroad, or from public or private research centers.
L'archive ouverte pluridisciplinaire HAL, est destinée au dépôt et à la diffusion de documents scientifiques de niveau recherche, publiés ou non, émanant des établissements d'enseignement et de recherche français ou étrangers, des laboratoires publics ou privés. 


\title{
Theory, simulation, and observation of discrete eigenmodes associated with lower hybrid solitary structures
}

\author{
P. W. Schuck, ${ }^{1}$ C. E. Seyler, ${ }^{2}$ J.-L. Pinçon, ${ }^{3}$ J. W. Bonnell, ${ }^{2}$ and
}

P. M. Kintner ${ }^{2}$

\begin{abstract}
A three-dimensional fluid description of nonlinear lower hybrid waves is investigated in the context of plasma density depletions. The objective is a basic understanding of lower hybrid solitary structures associated with transverse ion acceleration in the topside auroral ionosphere. The equations are linearized about a parabolic density depletion and solved. The solution consists of potential structures (eigenfunctions) which rotate in angle about the center of the density depletion. The eigenfrequencies are discrete for $|\omega|<\omega_{\text {LH }}$. These eigenfunctions rotate in a left-handed sense about the geomagnetic field and the solutions fall off exponentially outside the density depletion. The eigenfrequencies are continuous for $|\omega|>\omega_{\mathrm{LH}}$ and $k_{z} \neq 0$ but become discrete for $k_{z}=0$ in agreement with previous two-dimensional results [Seyler, 1994]. Simulations of the full nonlinear system are performed, and rotating eigenmodes are extracted from the spectrum. The results agree with the analytic results obtained from the linearized equations. The spectral properties of a lower hybrid solitary structure from the TOPAZ III sounding rocket are reexamined and found to be consistent with theoretical predictions for lower hybrid waves trapped within a density depletion as presented herein. A local wavelet frequency-wavenumber spectrum is constructed from data taken by moving an interferometer through a simulation at a typical rocket velocity. The results compare favorably with the local frequency-wavenumber spectrum of a lower hybrid solitary structure observed by the TOPAZ III sounding rocket.
\end{abstract}

\section{Introduction}

Lower hybrid solitary structures (LHSS) have presented a theoretical challenge since their identification by Labelle et al. [1986] on the MARIE sounding rocket. Frequently, bursts of transversely accelerated ions (TAI) are observed concurrently with LHSS [Arnoldy et al., 1992; Kintner et al., 1992; Vago et al., 1992; Lynch et al., 1996]. This suggested that LHSS may be a source of the ion conic distribution functions observed in the magnetosphere and ionosphere [Shelley et al., 1976; Sharp et al., 1977; Klumpar, 1979; Horowitz, 1980].

The MARIE observations identified the LHSS phenomena (termed "spikelets" by Labelle et al. [1986]) and noted coincidental evidence of TAI in the same altitude range. However, because of instrumental limitations this payload was unable to demonstrate a direct relationship between LHSS and the TAI. The same

\footnotetext{
${ }^{1}$ Laboratory of Plasma Studies, Cornell University, Ithaca, New York. '2 School of Electrical Engineering, Cornell University, Ithaca, New York. ${ }^{3}$ Centre National de la Recherche Scientifique, Orleans, France.
}

Copyright 1998 by the American Geophysical Union.

Paper number 97JA02922.

0148-0227/98/97JA-02922\$09.00 phenomena was also observed in the TOPAZ II data (Topside Probe of the Auroral Zone). This payload was also unsuccessful in demonstrating a correspondence between LHSS and TAI. The TOPAZ III sounding rocket [Arnoldy et al., 1992; Kintner et al., 1992; Vago et al., 1992] provided the first comprehensive analysis of LHSS and TAI. These observations were able to demonstrate a definitive correspondence between LHSS and the observations of TAI.

Interferometric analysis [Vago et al., 1992] of the wave data showed that the wavelength of the waves decreased within the LHSS and the phase angle-frequency relationship had characteristics similar to the theoretical dispersion relation for homogeneous lower hybrid (LH) waves. Additionally, the interferometric analysis demonstrated that the wave power extended below the normally sharp cutoff of the ambient lower hybrid resonance (LHR) within the LHSS events. This indicated that waves were propagating below the ambient LHR within the LHSS event. Because of the limitations of the cross-Fourier spectral methods used, it was impossible to differentiate between geophysical and payload generated waves occurring in the same frequency regime.

The Langmuir probe responded dramatically to several of the LHSS events recording large drops in the 
probe current. This response suggested deep density depletions which in some cases approached an $80 \%$ change measure relative to the background. However, questions regarding the validity of the Langmuir probe measurements have been raised by subsequent observations [Ergun et al., 1994, 1995]. Similar phenomena have also been observed by the Viking satellite [Block et al., 1987; Pottelette et al., 1988, 1992] at an altitude of 5800-6000 $\mathbf{k m}$ in the source region for auroral kilometric radiation (AKR). However, in this case the bursts of broadband electrostatic noise were correlated with the gradients of small-scale $(10 \mathrm{~km})$ density enhancements. The Freja [Eriksson et al., 1994; Dovner et al., 1994] satellite reported a direct correlation between LHSS and density depletions of $1-10 \%$ at an altitude of $1700 \mathrm{~km}$. Additionally, multiprobe measurements of the plasma density demonstrated that the plasma depletions were stationary in the plane normal to the magnetic field with respect to the ambient plasma. Collectively, these observations indicate that LHSS are a robust phenomena occurring in the nightside aurora in a height range from 500 to $6000 \mathrm{~km}$ and the events are conspicuously associated with density gradients.

Several theories have been proposed for the origin and evolution of the LHSS and the associated TAI. Chang and Coppi [1981] demonstrated that lower hybrid waves are capable of producing transversely accelerated ions. This mechanism requires an energetic (at least $1 \mathrm{eV}$ ) ion population for efficient resonant interaction with the high phase velocity lower hybrid waves excited by auroral electrons. Unfortunately, the density of energetic ions in typical auroral plasmas is too small to account for the observed fluxes of TAI [Retterer et al., 1986; Chang, 1993]. Retterer et al. [1986] proposed a nonlinear two time-scale model capable of efficiently heating low energy ion populations with linearly excited LH waves. This one-dimensional model described the coupling of high-frequency lower hybrid oscillations to a slow quasineutral density response similar to the $Z a$ kharov [1972] equations which describe the dynamics of coupled Langmuir and ion acoustic waves. This mechanism produces short wavelength, low phase velocity modes through the parametric decay of a monochromatic LH pump wave.

The two timescale analysis was extended to two and three dimensions by Shapiro et al. [1993,1995] based on the collapse theories of Musher and Sturman [1975] and Sotnikov et al. [1978]. The collapse theories predict that LH wave packets are modulationally unstable in several dimensions and will undergo a nonlinear cascade to high spatial wavenumber until the process is arrested at the dissipative length scale through a Landau resonance with the ions. The lower hybrid waves are nonlinearly focused into the quasineutral density depletions (or enhancements). This intensifies the local electric field enhancing the ponderomotive pressure. The ponderomotive pressure enhances the quasineutral density fluctuation leading to positive feedback or self-focusing of the LH waves. This mechanism leads to a complete description of the quasineutral density fluctuations and the strong electric field enhancement.

Although initial experimental observations seemed to be consistent with the collapse of LH wave packets [Chang, 1993] there appear to be difficulties with the collapse theories. First, the Musher and Sturman [1975] equations are based on the balance between the parallel electron ponderomotive force and the slow-mode electric field and pressure. Therefore these equations are inapplicable in two dimensions perpendicular to the magnetic field [Seyler, 1994] but may describe the collapse of LH waves in quasi-two dimensions [Tam and Chang, 1995]. A statistical analysis of LHSS observed by the Freja satellite [Pécseli et al., 1996] concluded that LH collapse was an unlikely scenario because of the pronounced discrepancies between the time and length scales predicted by the collapse models and those observed in the data. Finally, Robinson et al. [1996] compared the density depletions observed by Freja, TOPAZ III, and Viking with quantitative calculations based on the balance between kinetic and ponderomotive pressures. Robinson et al. [1996] concluded that the predictions of the collapse models were inconsistent with the observed depth of the density depletions. Furthermore, the collapse models predict density enhancements and depletions with equal probability, whereas only depletions were observed by Freja and TOPAZ III.

Recent analysis of data collected by the Auroral Microphysics and Ion Conics: Investigation of Space and Time (AMICIST) [Pinçon et al., 1997] and Physics of Auroral Zone Electrons II (PHAZE II) (Bonnell et. al. submitted manuscript 1997) sounding rockets has provided new details about the electric field structure of LHSS. A local frequency-wavenumber spectral estimation for the nine LHSS events observed on AMICIST was constructed. After dividing an event into two equal time segments, Pinçon et al. [1997] determined that the component of phase velocity parallel to the interferometer reversed direction as the rocket crossed the center of the event. This demonstrated that these LHSS were composed of wave modes with frequencies above the ambient LHR which rotated in a right-handed sense about the magnetic field. These observations are consistent with a model (presented in section 2.1 of this paper) of nonlinear LH phenomena. Additionally, this model predicts that for frequencies below the ambient LHR, LH waves in a density depletion rotate in a lefthanded sense about the magnetic field. Unfortunately, these wave modes were corrupted by payload emissions on the AMICIST flight. However, preliminary analysis (Bonnell et. al. submitted manuscript 1997) of the PHAZE II sounding rocket has determined the absolute sense of rotation of wave modes below the LHR. These results indicate that wave modes below the LHR rotate in a left-handed sense about the magnetic field and wave 
modes above the LHR rotate in a right-handed sense about the magnetic field in agreement with the theoretical predictions and AMICIST observations.

This paper will examine the properties of the electrostatic modes which exist below the LHR in LHSS. Section 2 presents a theoretical model for nonlinear $\mathrm{LH}$ phenomena. This three-dimensional model is the basis for the results discussed here and in the work of Pinçon et al. [1997]. The equations are linearized about a parabolic density depletion and the eigenfunctions are found. The eigenfunctions which have a frequency above the lower hybrid resonance (LHR) extend to infinity and the potential structures rotate in a right-handed sense about the magnetic field. The eigenfunctions which have a frequency below the LHR are "trapped" within the density depletion and the potential structure rotates in a left-handed sense about the magnetic field. Section 3 discusses numerical simulations of the full nonlinear system. Several individual eigenfunctions are examined by filtering the frequency spectrum. The numerical nonlinear behavior is in agreement with the linear normal mode description. Finally, section 4 reexamines event I from the TOPAZ III sounding rocket [Vago et al., 1992]. Using a fast wavelet transform technique [Pinçon et al., 1997], the local frequency-wavenumber spectrum of this event is compared with the spectrum constructed from an interferometer moving through a numerical simulation at a typical rocket velocity.

\section{Theory of Rotating LHSS}

The fundamental equations are the two-fluid equations with cold ions and isotropic, isothermal electrons. This complicated description is considerably simplified by making approximations based on the timescale and length scale of interest. The observed signature of lower hybrid solitary structures (LHSS) is a burst of waves near the local lower hybrid resonance (LHR). The associated electric field is predominantly perpendicular to the geomagnetic field $\vec{B}=B_{0} \hat{z}$ and has a wavelength much shorter than the ambient electromagnetic skin depth $\lambda_{e}=c / \omega_{\text {pe }}$, where $c$ is the speed of light and $\omega_{\text {pe }}$ is the electron plasma frequency. The short wavelength nature of the electric fields suggests that the LHSS are predominantly an electrostatic phenomena. Therefore the electromagnetic effects considered by Seyler [1994] are neglected.

\subsection{Electrostatic Model of LHSS}

The angular frequency of interest for LHSS is approximately the magnetized lower hybrid angular frequency $\Omega=\sqrt{\Omega_{\mathrm{ci}} \Omega_{\mathrm{ce}}}$, where $\Omega_{\mathrm{ci}}$ is the ion cyclotron frequency and $\Omega_{\mathrm{ce}}$ is the electron cyclotron frequency. The perpendicular spatial scales associated with LHSS phenomena are on the order of the ion sound gyroradius $\rho_{\mathrm{s}}=\sqrt{k T_{e} / m_{\mathrm{i}}} / \Omega_{\mathrm{i}}$, where $T_{\mathrm{e}}$ is the electron tempera- ture and $m_{\mathrm{i}}$ is the ion mass. These scales are short by comparison to the inferred parallel length of the structures which is certainly few hundred times $\rho_{8}$ and probably a few thousand times $\rho_{\mathrm{s}}$ [Vago et al., 1992]. Additionally, lower hybrid waves propagate nearly perpendicular to the magnetic field $\left(k_{\mathrm{z}} / k_{\perp} \sim \sqrt{m_{\mathrm{e}} / m_{\mathrm{i}}}\right)$. This justifies the use of a reduced description where the gradients parallel to the magnetic field are neglected in comparison to gradients perpendicular to the magnetic field. However, the parallel gradient of the electron velocity in the continuity equation and the parallel gradient of the $\phi$ in the electron momentum equation are retained to allow the propagation of lower hybrid waves along the magnetic field.

The ions are essentially unmagnetized, compressible, linear, and cold on these scales and may be described by a velocity potential $\vec{u}_{\mathrm{i}}=\nabla_{\perp \chi}$. These approximations yield the following equations for the evolution of the ions:

$$
\begin{aligned}
\partial_{t} n_{\mathrm{i}} & =-n_{0} \nabla_{\perp}^{2} \chi \\
\partial_{t} \chi & =-\frac{e}{m_{\mathrm{i}}} \varphi
\end{aligned}
$$

where $\varphi$ is the electrostatic potential $(\vec{E}=-\nabla \varphi)$ and $n_{0}$ is the homogeneous plasma density.

The electrons are magnetized on these scales and the perpendicular motion of the electron fluid is dominated by the electric drift across the magnetic field, modified by polarization corrections

$$
\begin{aligned}
\vec{u}_{\mathrm{e} \perp}= & \frac{\hat{z} \times \nabla_{\perp} \phi}{B_{0}} \\
& +\frac{1}{\Omega_{c e} B_{0}}\left[\partial_{t} \nabla_{\perp} \phi+\left(\frac{\hat{z} \times \nabla_{\perp} \phi}{B_{0}} \cdot \nabla_{\perp}\right) \nabla_{\perp} \phi\right],
\end{aligned}
$$

where

$$
\phi=\varphi-\frac{\gamma_{\mathrm{e}} k_{B} T_{\mathrm{e}}}{n_{0} e} n_{\mathrm{e}}
$$

and $n_{\mathrm{e}}$ is the electron density. The parallel electron momentum equation

$$
\partial_{t} v+\frac{\hat{z} \times \nabla_{\perp} \phi}{B_{0}} \cdot \nabla_{\perp} v=\frac{e}{m_{\mathrm{e}}} \partial_{z} \phi
$$

is necessary for the correct representation of three-dimensional lower hybrid waves. The parallel electron inertia is as important as the ion inertia when $k_{z} / k_{\perp} \geq \sqrt{m_{\mathrm{e}} / m_{\mathrm{i}}}$. Under these approximations the electron density evolves according to the continuity equation

$$
\partial_{t} \Gamma+\frac{\hat{z} \times \nabla_{\perp} \phi}{B_{0}} \cdot \nabla_{\perp} \Gamma=-n_{0} \partial_{z} v
$$

where only the leading order nonlinearity has been retained and

$$
\Gamma=n_{\mathrm{e}}+\frac{m_{\mathrm{e}} n_{0}}{e B_{0}^{2}} \nabla_{\perp}^{2} \phi
$$


is the density of electron guiding centers modified by polarization corrections. The dynamics of the two fluids are coupled through the electrostatic potential which is determined from a reduced Poisson's equation

$$
\nabla_{\perp}^{2} \varphi=\frac{e}{\epsilon_{0}}\left(n_{\mathrm{e}}-n_{\mathrm{i}}\right)
$$

Again, the parallel gradients have been neglected to reflect the reduced geometry appropriate to the propagation of lower hybrid waves. This approximation also eliminates the higher-frequency phenomena associated with Langmuir waves. The electron continuity (1e) and Poisson's equation (1f) correct the quasineutral electromagnetic model [Seyler, 1994] for nonneutral effects associated with propagation in an subcritical plasma where $\omega_{\mathrm{pe}}<\Omega_{\mathrm{ce}}$. Linearization of (1) within a homogeneous infinite plasma yields the lower hybrid dispersion relation

$$
\omega^{2} \approx \omega_{\mathrm{LH}}^{2}\left(1+R k_{\mathrm{z}}^{2} / k_{\perp}^{2}\right)
$$

where

$$
\omega_{\mathrm{LH}}^{2}=\frac{\Omega_{\mathrm{ce}}^{2} \omega_{\mathrm{pi}}^{2}}{\Omega_{\mathrm{ce}}^{2}+\omega_{\mathrm{pe}}^{2}}
$$

is the lower hybrid resonance, $\omega_{\mathrm{pi}}$ is the ion plasma frequency, $\omega_{\mathrm{pe}}$ is the electron plasma frequency, $R=$ $m_{\mathrm{i}} / m_{\mathrm{e}}, k_{\mathrm{z}}$ is the wave vector along the magnetic field, and $k_{\perp}$ is the wave vector in the plane normal to the magnetic field.

\subsection{Rotating Eigenmodes in a Density Depletion}

Subsequent sections examine the three-dimensional rotating eigenmodes of cylindrical density depletion with a parabolic profile. Seyler [1994] considered the twodimensional eigenmodes and found bound states for frequencies above and below the lower hybrid frequency. This anomalous behavior is due to the resonant nature of two dimensional LH oscillations and is rectified by considering finite wavenumbers parallel to the magnetic field. The equations of section 2.1 are simplified by considering a cold plasma.

$$
\begin{aligned}
\partial_{t} n_{\mathbf{i}} & =-n_{0} \nabla_{\perp}^{2} \chi, \\
\partial_{t} \chi & =-\frac{e}{m_{\mathbf{i}}} \varphi, \\
\partial_{t} \Gamma+\frac{\hat{z} \times \nabla_{\perp} \varphi}{B_{0}} \cdot \nabla_{\perp} \Gamma & =-n_{0} \partial_{z} v, \\
\partial_{t} v+\frac{\hat{z} \times \nabla_{\perp} \varphi}{B_{0}} \cdot \nabla_{\perp} v & =\frac{e}{m_{\mathrm{e}}} \partial_{z} \varphi, \\
\nabla_{\perp}^{2} \varphi & =\frac{e}{\epsilon_{0}}\left(n_{\mathrm{e}}-n_{\mathbf{i}}\right),
\end{aligned}
$$

where

$$
\Gamma=n_{\mathrm{e}}+\frac{m_{\mathrm{e}} n_{0}}{e B_{0}^{2}} \nabla_{\perp}^{2} \varphi
$$

The polarization correction to the density is dependent only on the electrostatic potential $\varphi$, in the cold approximation. This cold electrostatic plasma assumption is quite good for perpendicular wavelengths greater than electron gyroradius and smaller than the electromagnetic skin depth.

The system is linearized about a cylindrically symmetric quasi-neutral equilibrium density of the form

$$
\Gamma=n_{\mathrm{i}}=n_{\mathrm{e}}= \begin{cases}n_{0}\left[1+\Delta\left(1-r^{2} / a^{2}\right)\right] & r<a, \\ n_{0} & r>a,\end{cases}
$$

where $\Delta<0$ is the depth of the equilibrium density profile relative to the background density. Assuming rotating solutions of the form

$$
\varphi(r, \theta, z, t)=\widehat{\varphi}(r) \exp \left[i\left(m \theta+k_{z} z-\omega t\right)\right]
$$

Equations (3) become

$$
\begin{aligned}
-i \omega n_{\mathrm{i}} & =-n_{0} \widehat{\nabla}_{\perp}^{2} \chi, \\
-i \omega \chi & =-\frac{e}{m_{\mathrm{i}}} \varphi, \\
-i \omega \Gamma+i \frac{m n_{0} \eta}{B_{0}} \varphi & =-i n_{0} k_{z} v, \\
-i \omega v & =i \frac{e}{m_{\mathrm{e}}} k_{z} \varphi, \\
\hat{\nabla}_{\perp}^{2} \varphi & =\frac{e}{\epsilon_{0}}\left(n_{\mathrm{e}}-n_{\mathrm{i}}\right),
\end{aligned}
$$

where (4) relates $\Gamma$ to $n_{\mathrm{e}}$ and $\varphi$,

$$
\begin{aligned}
\hat{\nabla}_{\perp}^{2} & =\frac{1}{r} \frac{d}{d r}\left(r \frac{d}{d r}\right)-\frac{m^{2}}{r^{2}} \\
\eta & = \begin{cases}2 \Delta / a^{2} & r<a, \\
0 & r>a .\end{cases}
\end{aligned}
$$

Equations (6) are combined to obtain the wave equation (Helmholtz equation)

$$
\left(\widehat{\nabla}_{\perp}^{2}+k^{2}\right) \hat{\varphi}=0
$$

or equivalently, Bessel's equation

$$
\frac{d^{2} \hat{\varphi}}{d r^{2}}+\frac{1}{r} \frac{d \hat{\varphi}}{d r}+\left(k^{2}-\frac{m^{2}}{r^{2}}\right) \hat{\varphi}=0
$$

The continuity of the frequency $\omega$ and parallel wave vector $k_{z}$ across $r=a$ implies that the eigenvalue $k^{2}$ must be discontinuous at $r=a$

$$
k^{2}= \begin{cases}\gamma^{2}=\frac{\omega_{\mathrm{LH}}^{2} R\left(k_{z}^{2}-2 \Delta m \omega / \Omega_{\mathrm{ce}} a^{2}\right)}{\omega^{2}-\omega_{\mathrm{LH}}^{2}} & r<a, \\ k_{\perp}^{2}=\frac{\omega_{\mathrm{LH}}^{2} R k_{z}^{2}}{\omega^{2}-\omega_{\mathrm{LH}}^{2}} & r>a .\end{cases}
$$

Equation (7c) defines the local dispersion relations inside and outside the density cavity. For $k^{2}>0$ the radial solution has wavelike (propagating) characteristics. For $k^{2}<0$ the radial solution has evanescent (decaying) behavior. Analysis of the full eigenvalue problem with boundary and continuity conditions determines the allowed eigenfrequencies. This treatment is deferred to section 2.4 . 


\subsection{Spectral Properties of LHSS}

The general spectral properties of LHSS are revealed through a plot of the radial eigenvalue $k^{2}$, versus $\omega$ in the interior $\left(k^{2}=\gamma^{2}\right)$ and exterior $\left(k^{2}=k_{\perp}^{2}\right)$ of the density depletion. A plot for the $m=1$ solutions is shown in Figure 1. The sense of rotation is determined from the relative signs of $\omega$ and $m$. Using (5) and choosing $m>0$, the sign of the frequency determines the direction of the azimuthal phase velocity: the negative frequency solutions rotate in a left-handed sense about the magnetic field and the positive frequency solutions rotate in a right-handed sense about the magnetic field. Both the interior and exterior solutions exhibit resonance behavior $\left(k^{2} \rightarrow \infty\right)$ at the LHR where there is distinct change in their character. It is convenient to refer to solutions with $\omega^{2}>\omega_{\mathrm{LH}}^{2}$ as "above the lower hybrid resonance" and solutions with $\omega^{2}<\omega_{\mathrm{LH}}^{2}$ as "below the lower hybrid resonance."

2.3.1. Above the LHR: $\omega^{2}>\omega_{\text {LH }}^{2}$. The exterior eigenvalue is always positive $\left(k_{\perp}^{2}>0\right)$ for $\omega^{2}>$ $\omega_{\mathrm{LH}}^{2}$. Therefore both left and right-handed solutions propagate outside the density depletion above the LHR. However, the interior eigenvalue is positive $\left(\gamma^{2}>0\right)$ only for positive frequencies $\omega>\omega_{\mathrm{LH}}$. Thus, above the LHR only right-handed waves propagate within the density depletion. Above the LHR the left-handed waves decay (evanesce) within the density depletion. This suggests that these mode should be interpretated as reflected states.

2.3.2. Below the LHR: $\boldsymbol{\omega}^{2}<\boldsymbol{\omega}_{\mathrm{LH}}^{2}$. The exterior eigenvalue is always negative $\left(k_{\perp}^{2}<0\right)$ for $\omega^{2}<\omega_{L H}$ and the solution is evanescent outside the depletion. However, the interior eigenvalue is positive $\left(\gamma^{2}<0\right)$ for

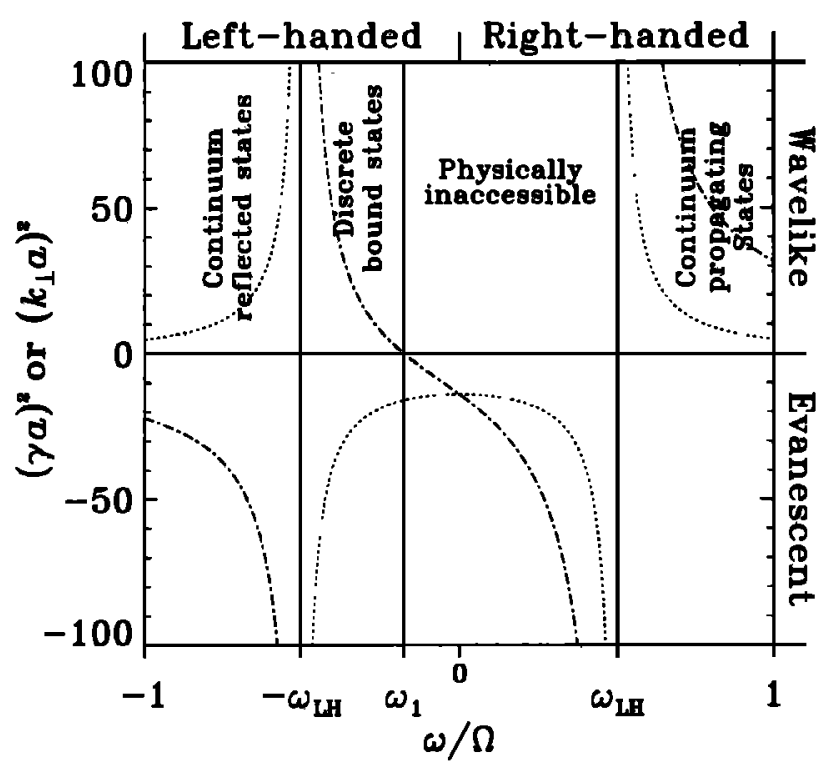

Figure 1. The function $\gamma^{2}(\omega)$ for $r<a$ (dotted-dashed line) and $k_{\perp}^{2}(\omega)$ for $r>a$ (dotted line) for $\Delta=-0.35$, $a=2.5 \rho_{\mathrm{s}}, R k_{z}=1.5 \pi / \rho_{\mathrm{s}}$, and $m=1$. Right-handed modes have $\omega>0$, and left-handed modes have $\omega<0$. negative frequencies which satisfy $-\omega_{\mathrm{LH}}<\omega<\omega_{m}<0$. Thus, below the LHR only left-handed waves may propagate within the density depletion. Below the LHR the right-handed waves decay both inside and outside the density depletion and are forbidden. Clearly, the spectrum of states which exist below the ambient LHR are left-handed and decay exponentially outside the density depletion and are bound states with finite energy per unit length along the magnetic field.

\subsection{Solution of the Eigenvalue Problem}

The discussion of the eigenvalue problem is restricted to density depletions $\Delta<0$ and $m>0$ for the sake of brevity. Equivalent results may be obtained for $m<$ 0 because the dispersion relations (7c) are invariant with respect to the transformation $(\omega, m) \Rightarrow(-\omega,-m)$ which preserves the sense of rotation about the magnetic field. A density enhancement $(\Delta>0)$ leads to an exchange of the results for left and right-handed waves since the dispersion relations are invariant with respect to the transformation $(\Delta, \omega, m) \Rightarrow(-\Delta,-\omega, m)$ which changes the sense of rotation about the magnetic field.

Because of the discontinuous nature of (7) at $r=a$, (7a) is solved independently in the two regions $r<a$ and $r>a$

$$
\widehat{\varphi}(r)= \begin{cases}A \mathrm{~J}_{m}(\gamma r)+B \mathrm{~N}_{m}(\gamma r) & r<a \\ C \mathrm{~J}_{m}\left(k_{\perp} r\right)+D \mathrm{~N}_{m}\left(k_{\perp} r\right) & r>a\end{cases}
$$

where $\mathrm{J}_{m}$ is a Bessel function of the first kind and $\mathrm{N}_{m}$ is a Neumann function or Bessel function of the second kind. The solution $\hat{\varphi}(r)$ and its first derivative $d \hat{\varphi}(r) / d r$ must be continuous across $r=a$ since (7a) is second order in $r$. Furthermore, the solution must be regular at the origin and zero at infinity. There are five parameters for the general solution: the four coefficients $A-D$ and the eigenfrequency $\omega$. Three of the coefficients and the eigenfrequency may by used to satisfy the boundary and continuity conditions. One of the coefficients $A-D$ remains undetermined because (7a) is homogeneous. Following the discussion in section 2.3 the spectrum divides neatly into scattering states where $\omega^{2}>\omega_{\mathrm{LH}}^{2}$ and bound states where $\omega^{2}<\omega_{\mathrm{LH}}^{2}$.

2.4.1. Scattering states $\omega^{2}>\omega_{\mathrm{LH}}^{2}$. For frequencies above the LHR the solution to (7) takes the form

$$
\hat{\varphi}(r)= \begin{cases}A \mathrm{~J}_{m}(\gamma r) & r<a, \\ C \mathrm{~J}_{m}\left(k_{\perp} r\right)+D \mathrm{~N}_{m}\left(k_{\perp} r\right) & r>a .\end{cases}
$$

Since both $\mathrm{J}_{m}$ and $\mathrm{N}_{m}$ satisfy the boundary condition $\hat{\varphi}(r \rightarrow \infty)=0$, the coefficients $C$ and $D$ may be used to satisfy the continuity conditions at $r=a$ :

$$
\frac{C}{A}=\frac{\gamma \mathrm{J}_{m}^{\prime}(\gamma a) \mathrm{N}_{m}\left(k_{\perp} a\right)-k_{\perp} \mathrm{J}_{m}(\gamma a) \mathrm{N}_{m}^{\prime}\left(k_{\perp} a\right)}{k_{\perp} \mathrm{J}_{m}\left(k_{\perp} a\right) \mathrm{N}_{m}^{\prime}\left(k_{\perp} a\right)-k_{\perp} \mathrm{J}_{m}^{\prime}\left(k_{\perp} a\right) \mathrm{N}_{m}\left(k_{\perp} a\right)}
$$

$$
\frac{D}{A}=-\frac{\gamma \mathrm{J}_{m}^{\prime}(\gamma a) \mathrm{J}_{m}\left(k_{\perp} a\right)-k_{\perp} \mathrm{J}_{m}(\gamma a) \mathrm{J}_{m}^{\prime}\left(k_{\perp} a\right)}{k_{\perp} \mathrm{J}_{m}^{\prime}\left(k_{\perp} a\right) \mathrm{N}_{m}\left(k_{\perp} a\right)-k_{\perp} \mathrm{J}_{m}\left(k_{\perp} a\right) \mathrm{N}_{m}^{\prime}\left(k_{\perp} a\right)}
$$


Clearly, the eigenfrequency is not needed to satisfy any of the continuity conditions and remains a free parameter. Thus the spectrum associated with LHSS for $\omega^{2}>\omega_{\mathrm{LH}}^{2}$ will be continuous. However, within the continuous spectrum there are discrete points of "spectral concentration" or "resonances" where the electric field is strongly enhanced in the interior of the density depletion. These resonances occur only for $\omega>$ $\omega_{\text {LH }} \Rightarrow\left(\gamma^{2}>0, k_{\perp}^{2}>0\right)$ and are right-hand rotating eigenmodes. For $\omega<-\omega_{\text {LH }} \Rightarrow\left(\gamma^{2}<0, k_{\perp}^{2}>0\right)$ the eigenmodes behave like $\widehat{\varphi} \propto \mathrm{J}_{m}(i|\gamma| r)$ in the interior of the density depletion and fall off rapidly as $r \rightarrow 0$. These states correspond to reflected waves and are lefthand rotating eigenmodes. A detailed discussion of the continuum eigenmodes is complicated and is deferred to a subsequent paper where the scattering problem will be treated in rigorous fashion.

2.4.2. Bound states $\omega^{2}<\omega_{\mathrm{LH}}^{2}$. For frequencies below the LHR the solution to (7) takes the form

$$
\hat{\varphi}(r)= \begin{cases}A \mathrm{~J}_{m}\left(\gamma_{m n} r\right) & r<a, \\ A \frac{\mathrm{J}_{m}\left(\gamma_{m n} a\right) \mathrm{K}_{m}\left(\kappa_{m n} r\right)}{\mathrm{K}_{m}\left(\kappa_{m n} a\right)} & r>a,\end{cases}
$$

where $\kappa^{2}=-k_{\perp}^{2}$. The continuity of $d \hat{\varphi} / d r$ requires that

$$
\begin{aligned}
& \gamma_{m n} \mathrm{~J}_{m}^{\prime}\left(\gamma_{m n} a\right) \mathrm{K}_{m}\left(\kappa_{m n} a\right) \\
& \quad-\kappa_{m n} \mathrm{~J}_{m}\left(\gamma_{m n} a\right) \mathrm{K}_{m}^{\prime}\left(\kappa_{m n} a\right)=0,
\end{aligned}
$$

where $m$ and $n$ are the azimuthal and radial eigenvalue indices. This transcendental relation will be satisfied only for "special" frequencies in the range $-\omega_{\text {LH }}<$ $\omega_{m n}<\omega_{m}<0 \Rightarrow\left(\gamma^{2}>0, k_{\perp}^{2}<0\right)$. This leads to discrete spectrum of left-hand rotating modes below the LHR where

$\frac{\omega_{m n}}{\omega_{\mathrm{LH}}}=-\frac{\omega_{\mathrm{LH}} \Delta m}{\Omega_{\mathrm{ci}} \gamma_{m n}^{2} a^{2}}-\sqrt{\left(1+\frac{k_{z}^{2}}{\gamma_{m n}^{2}}\right)+\left(\frac{\omega_{\mathrm{LH}} \Delta m}{\Omega_{\mathrm{ci}} \gamma_{m n}^{2} a^{2}}\right)^{2}}$

These modes fall off exponentially as $r \rightarrow \infty$ and correspond to bound states.

The two-dimensional results of Seyler [1994] are recovered from this model in the limit $k_{z} \rightarrow 0$. For $k_{z}=0$, the eigenfunctions for both (9) and (10) become

$$
\hat{\varphi}(r)= \begin{cases}A \mathrm{~J}_{m}\left(\gamma_{m n} r\right) & r<a, \\ A \mathrm{~J}_{m}\left(\gamma_{m n} a\right)\left(\frac{r}{a}\right)^{-m} & r>a,\end{cases}
$$

where

$$
\mathrm{J}_{m-1}\left(\gamma_{m n} a\right)=0
$$

determines the allowed eigenfrequencies

$$
\frac{\omega_{m n}^{ \pm}}{\omega_{\mathrm{LH}}}=-\left[\frac{\omega_{\mathrm{LH}} \Delta m}{\Omega_{\mathrm{ci}} \gamma_{m n}^{2} a^{2}} \pm \sqrt{1+\left(\frac{\omega_{\mathrm{LH}} \Delta m}{\Omega_{\mathrm{ci}} \gamma_{m n}^{2} a^{2}}\right)^{2}}\right]
$$

Here the plus sign corresponds to left-hand modes and the minus sign corresponds to right-hand modes.

\subsection{Discussion of Theory}

These results indicate that the observed spectrum within LHSS should consist of a continuous spectrum of right-handed modes above the LHR and a discrete spectrum of left-handed modes below the LHR. The continuum contains points of spectral concentration (resonances) which result in an enhanced electric field within the density depletion. These three-dimensional resonances are vestiges of the two-dimensional bound states found above the LHR by Seyler [1994]. The continuous spectrum above the LHR may propagate outside the density depletion whereas discrete spectrum below the LHR should be strongly localized within the density depletion. The discrete bound states (solid circles) and the resonances (open circles) in the continuum are shown in Figure 2 for the parameters associated with the simulation discussed in section 3 and the experimental observations discussed in section 4. Both the resonances and bound states approach the LHR as either the azimuthal $m$, or radial $n$, indices goes to infinity. Thus the resonance behavior of homogeneous LH modes is reproduced by the cavity eigenmodes as $\omega^{2} \rightarrow \omega_{\mathrm{LH}}^{2}$. However, for low values of $n$, the eigenfrequency of the bound states actually decreases and $n$ increases. This interesting "dispersive" property of the discrete bound states will be addressed again in section 4 .

Observe in Figure 1, that the interior eigenvalue $\gamma^{2}$ may be an order of magnitude larger that the exterior eigenvalue $k_{\perp}^{2}>0$ for the same frequency. This suggests that a linear mechanism may be responsible for the short-wavelength large-amplitude electric fields associated with LHSS. Both of these observations may be explained through the scattering of long wavelength VLF hiss off preexisting density depletions which results in the excitation of resonant cavity eigenmodes. However, the electrostatic bound states must be excited other mechanisms. Ergun et al. [1995] has postulated that bound or "trapped" states may be formed by ducting $\mathrm{LH}$ waves down field-aligned density depletions. Under this scenario, LH waves are produced just above the LHR by precipitating electrons [Maggs, 1976]. These waves propagate earthward and encounter the density depletion which ducts the waves to lower altitudes where the ambient LHR is above the wave frequency. This senerio is not applicable to LHSS within the context of the model described in this paper. It may be demonstrated using (7c) that left-handed LH waves above the ambient LHR are reflected $\left(\gamma^{2}<0\right)$ from the density depletion. Therefore these waves cannot be ducted to lower altitudes to form bound states. Bound states may be formed by local plasma fluctuations within the density depletion or by resonant coupling to long wavelength electromagnetic magnetosonic fluctuations propagating outside the density depletion.

The effect of the density depletion on the LH dispersion relation can be understood by considering the degeneracy associated with propagation in the homo- 


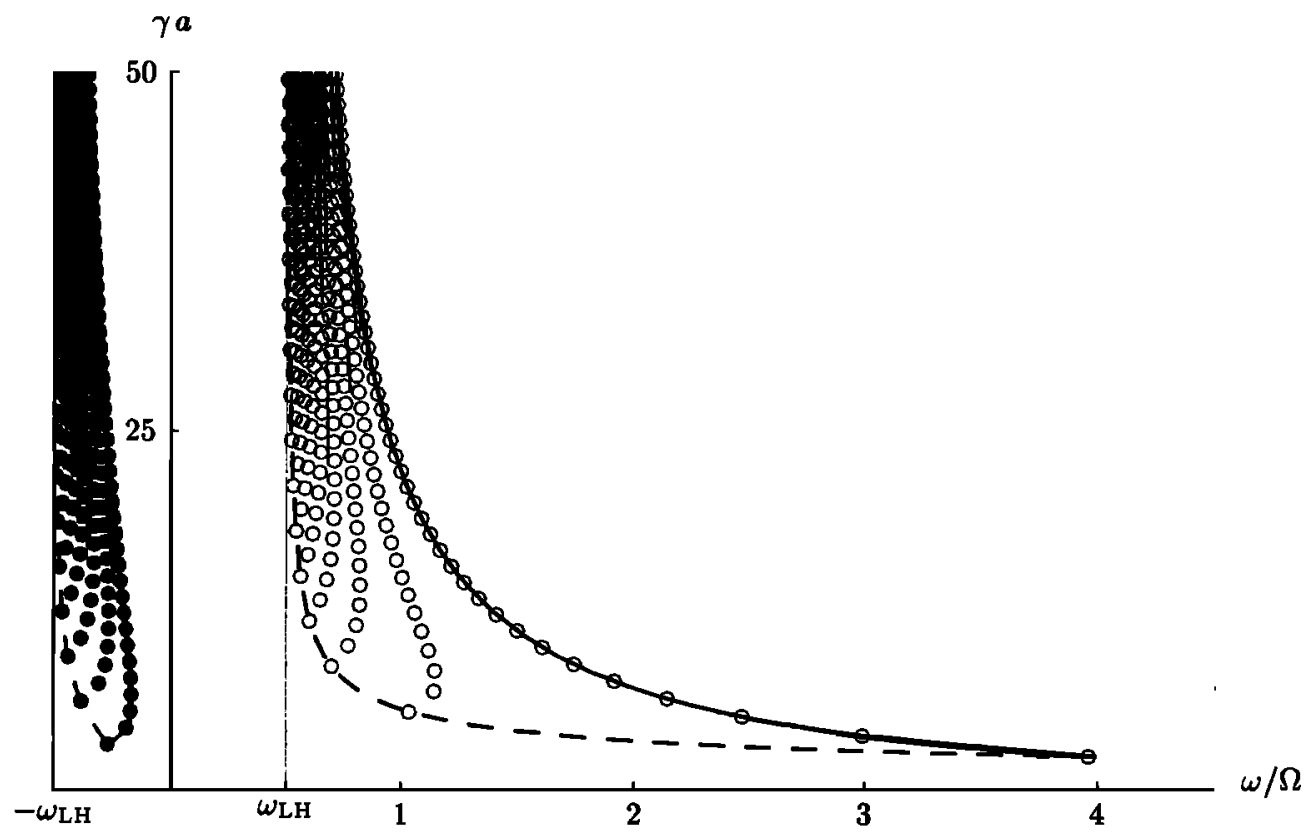

Figure 2. Discrete bound states (solid circles) and resonances (open circles) in the continuum of a LHSS with $\Delta=-0.35, a=2.5 \rho_{\mathrm{s}}$, and $R k_{z}=1.5 \pi / \rho_{\mathrm{s}}$. The solid line connects the radial eigenvalues $n$ for $m=1$. The dashed line connects the azimuthal eigenvalues $m$ for $n=1$.

geneous infinite plasma. The choice of basis functions in a homogeneous plasma is not limited to plane waves $\phi \propto \exp (\vec{k} \cdot \vec{x}-\omega t)$. The cylindrical basis functions $\phi \propto \mathrm{J}_{m}\left(k_{\perp} r\right) \exp \left[i\left(m \theta+k_{\mathrm{z}} z-\omega t\right)\right]$ also furnish an appropriate representation of lower hybrid waves. Here $m$ is the azimuthal eigenvalue which may take on only integral values. This basis decomposes the lower hybrid fluctuations into traveling waves which rotate in left-handed or right-handed sense about the magnetic field depending on the relative signs of $\omega$ and $m$. The corresponding dispersion relation for the cylindrical basis functions is independent of the azimuthal eigenvalue $m$ and remains (2). Thus both left-handed and right-handed modes rotate with the same phase velocity in the homogeneous infinite plasma. The density depletion breaks this degeneracy by introducing an explicit dependence on the azimuthal eigenvalue $m$ to the interior dispersion relation $\left(\gamma^{2}\right)$. This perturbation permits left-handed lower hybrid waves to propagate within the density depletion at frequencies below the ambient LHR. In fact, the interaction of the rotating mode with the density gradient in the depletion allows propagation of LH waves below the minimum LHR in the density depletion. For a modest density depletion $(|\Delta| \ll 1)$ the LHR at the center of the cavity is

$$
\omega_{R}(0) \approx \omega_{\mathrm{LH}}\left(1+\frac{\Delta}{2} \frac{\Omega_{\mathrm{ce}}^{2}}{\omega_{\mathrm{pe}}^{2}+\Omega_{\mathrm{ce}}^{2}}\right)+\mathcal{O}\left(\Delta^{2}\right) .
$$

The frequency shift due to the density gradient for a two-dimensional eigenfunction is larger than the maximum change in the local LHR if

$$
\frac{m}{\gamma_{m n}^{2} a^{2}}>\frac{1}{2} \sqrt{\frac{m_{\mathrm{e}}}{m_{\mathrm{i}}}} \frac{\Omega_{\mathrm{ce}}^{2}}{\omega_{\mathrm{pe}}^{2}} \geq \frac{1}{2} \frac{\omega_{\mathrm{LH}} \Omega_{\mathrm{ce}}}{\omega_{\mathrm{pe}}^{2}} .
$$

Note that for the $m=1, n=1$ eigenfunction $\gamma_{11} a=$ $2.401 \cdots$ [Abramowitz and Stegun, 1964]. For ambient plasma conditions during the TOPAZ III flight $\Omega_{\mathrm{ce}}^{2} / \omega_{\mathrm{pe}}^{2} \approx 3$. Clearly, the shift due to the density gradient dominates the correction due to the local LHR for the lowest lying eigenstates.

These rotating eigenmodes are not to be confused with electrostatic drift waves. Although, both types of waves propagate essentially perpendicular to both the equilibrium density gradient and the magnetic field, the wave dynamics are completely different. Drift waves require a diamagnetic current produced by the drift of thermal electrons perpendicular to the density gradient. This is clearly absent in the results obtained section $\mathbf{2 . 2}$ for a cold plasma. The physics of LH waves is greatly modified by the Hall current which results from the disparate response of magnetized electrons and unmagnetized ions.

To illustrate the importance of the Hall current in the context of lower-hybrid waves, consider a slab geometry with lower hybrid waves propagating in the $\hat{\mathbf{x}}$ direction within a density gradient along $\hat{\mathbf{y}}$. This problem will be treated in the high-density limit where the transverse electron polarization current dominates the displacement current. Therefore the quasi-neutrality asummption is valid and $\nabla \cdot \mathbf{J}=0$ may be used to determine the electrostatic potential. Using (1a)-(1c) and $\nabla \cdot \mathbf{J}=0$, the electrostatic potential is found to satisfy 


$$
\left[\partial_{t}^{2} \partial_{x}^{2}+\Omega^{2} \partial_{x}^{2}+\left(\Omega_{\mathrm{ce}} / L_{n}\right) \partial_{t} \partial_{x}\right] \varphi=0
$$

The Hall term is $\left(\Omega_{\mathrm{ce}} / L_{n}\right) \partial_{t} \partial_{x}$ and $L_{n}=n_{0} /\left(\partial_{y} n_{0}\right)$ is the scale length of the equilibrium density gradient. The Hall term produces a frequency shift away from the LHR which is observed as a difference in phase velocity between right and left propagating waves. The shift in phase velocity is given by the Hall velocity $v_{\mathrm{H}}=\Omega_{\mathrm{ce}} / k^{2} L_{n}$. The local dispersion relation resulting from (15) is

$$
\omega \approx-\frac{k v_{\mathrm{H}}}{2} \pm \Omega \text {. }
$$

This local dispersion relation explicitly shows how the Hall velocity breaks the degeneracy between left and right propagating waves. It should emphasized that the local approximation is not valid for small $k$, and the problem should be treated as an eigenvalue problem. However, frequency shift due to the Hall velocity may be estimated as follows: Let $L_{n}^{-1}=\Delta / a$, where $\Delta$ is the relative change in the density across the gradient and a is the width of the change in the density. The frequency shift is given by $\delta \omega=-k v_{\mathrm{H}} / 2=-\Delta \Omega_{\mathrm{ce}} / 2 k a$. Taking $\Delta=-0.01, a=10 \mathrm{~m}, k a=2 \pi$, and $\Omega_{\mathrm{ce}}=6 \times 10^{6} \mathrm{~s}^{-1}$, the frequency shift is found to be $\delta f \approx 800 \mathrm{~Hz}$. Assuming an $\mathrm{O}^{+}$plasma, the magnetized lower hybrid frequency $f_{\mathrm{LH}}$, is $5.6 \mathrm{kHz}$ for these parameters. Clearly, even a $1 \%$ density change over $10 \mathrm{~m}$ results in an observable shift in the frequency for right and left propagating waves. The wavenumber dependence of the Hall velocity shows that this effect is more important for long wavelength modes which is evident in Figure 2.

A similar Hall velocity plays a fundamental role in electron magnetohydrodynamics, which has been discussed previously in beam injection problems and in plasma opening switches [Kingsep et al., 1984; Rudakov et al., 1991]. It was noted that magnetic flux can penetrate collisionless plasma normal to the equilibrium density gradient with velocity $v_{\mathrm{H}}=\Omega_{\mathrm{ce}} \lambda_{\mathrm{e}}^{2} / L_{n}$, where $\lambda_{e}$ is the collisionless skin depth [Gordeev and Rudakov, 1970]. The difference between this flux penetration Hall velocity and the Hall velocity for lower hybrid waves is the factor $k^{2} \lambda_{\mathrm{e}}^{2}$ due to transverse electron inertia from the electron polarization drift and the absence of electromagnetic effects in the electrostatic model.

\section{Simulation of Rotating LHSS}

The full three-dimensional dynamical system in (1) was solved using a mixed spectral and finite difference algorithm on a $N_{x} \times N_{y} \times N_{z}=80 \times 80 \times 4$ grid. The four grids in the parallel direction were sufficient to resolve the fundamental parallel wavenumber used for all of the eigenfunctions. The time advance is based on techniques developed for the reduced magnetohydrodynamic equations [Longcope, 1993]. The algorithm combines psuedo leapfrog [Forest and Ruth, 1990] scheme for the linear terms with a second order Runge-Kutta scheme for the nonlinear terms. The nonlinearities in the transverse plane were calculated using standard psuedo spectral techniques [Orszag, 1971] with a generalized prime factor algorithm (GPFA) [Temperton, 1992] fast Fourier transform algorithm. Partial dealiasing was implemented by truncating the spectral representations outside the cylindrical region [Orszag, 1971] $k_{z}^{2}+k_{y}^{2}=8 / 9 \times\left(N_{x} / 2\right)^{2}$ and calculating the nonlinear terms on a shifted grid during the second phase of the psuedo leapfrog time advance [Seyler, 1990].

The linear kinetic dispersion relation for the LH surface was calculated using WHAMP (Waves in Homogeneous Anisotropic Multicomponent Plasma) [Ronnmark, 1982] for the ambient plasma parameters associated with event I discussed in section 4: $B_{0}=0.36$ $\mathrm{G}$, and ion densities $n_{\mathrm{O}^{+}}=3.9 \times 10^{3} \mathrm{~cm}^{-3}, n_{\mathrm{H}^{+}}=$ $350 \mathrm{~cm}^{-3}$ and $n_{\mathrm{He}^{+}}=60 \mathrm{~cm}^{-3}$. Additionally, the temperature relations $T_{\mathrm{O}^{+}}=T_{\mathrm{H}^{+}}=T_{\mathrm{He}^{+}}=T_{\mathrm{e}}=0.3$ $\mathrm{eV}$ were assumed. A comparison of the kinetic dispersion relation and the electrostatic model discussed in section 2 is shown in Figure 3. The contours of constant frequency are separated by $0.5 \omega_{\mathrm{LH}}$. The abscissa is in units of $k_{\perp} \rho_{\mathrm{O}}+$ and the ordinate is in units of $k_{z} \rho_{\mathrm{O}+}$. The domain of wave vectors spanned by the simulation region $(51 \mathrm{~m}$ by $51 \mathrm{~m}$ perpendicular to the magnetic field and $1.8 \mathrm{~km}$ along the magnetic field) is shaded in grey and corresponds to the plateau of the dispersion surface for these parameters. The kinetic dispersion surface has negligible damping over the wavenumbers shown except just above the simulation region $\left(k_{z} \rho_{\mathrm{O}}+\sim 0.1\right)$ where the LH surface becomes

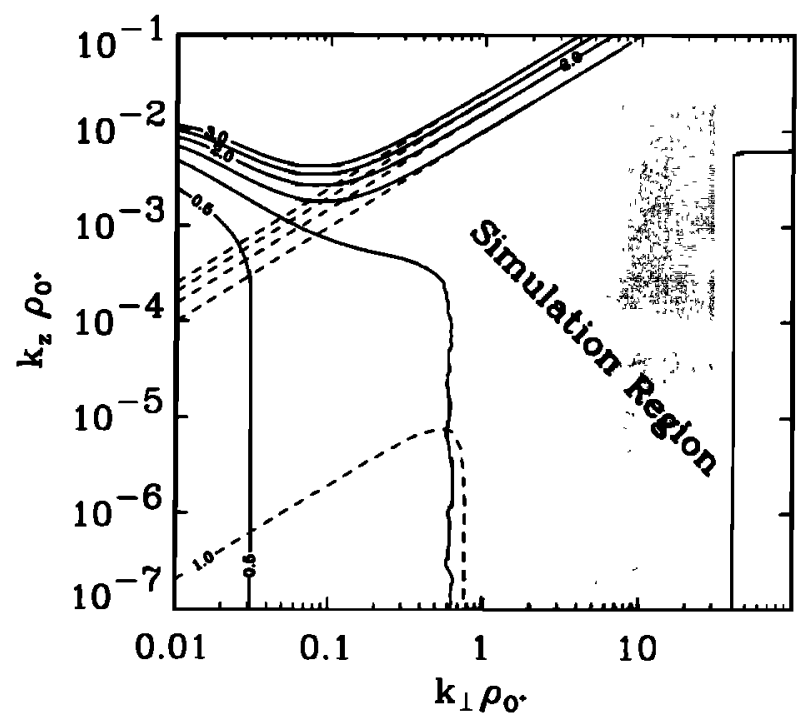

Figure 3. A comparison of the kinetic homogeneous linear dispersion relation (solid line) and the electrostatic model (dashed line) discussed in section 2 for ambient plasma conditions associated with event I on the TOPAZ III flight (see section 4). The contours of constant frequency are separated by $0.5 \omega_{\text {LH }}$. The abscissa is in units of $k_{\perp} \rho_{\mathrm{O}^{+}}$, and the ordinate is in units of $k_{z} \rho_{\mathrm{O}^{+}}$. The domain of wave vectors spanned by the simulation region is shaded in grey. 
structured by the hydrogen Bernstein modes. The electrostatic model reproduces the $\mathrm{LH}$ resonance cone over a wide range of perpendicular wave numbers $k_{\perp} \rho_{\mathrm{O}^{+}}=$ $0.5-40$. The model dispersion relation strays from the kinetic dispersion relation at $k_{\perp} \rho_{\mathrm{O}^{+}} \approx 0.5$. This deviation may be attributed to the electrostatic nature of the model. Electromagnetic corrections become significant for wavelengths approaching the electromagnetic skin depth $\lambda_{e} \approx 81 \mathrm{~m}$. This critical wavenumber occurs at $k_{\perp} \lambda_{\mathrm{e}}=1$ which corresponds to $k_{\perp} \rho_{\mathrm{O}^{+}} \approx 0.5$ on the plot. The fundamental perpendicular wavenumber of the simulation region was about $k_{\perp} \rho_{\mathrm{O}+} \approx 0.8$. Therefore electromagnetic corrections were negligible.

The simulation was initialized with an equilibrium $n_{\mathrm{e}}=n_{\mathrm{i}}$ smoothed parabolic density profile. The density depletion was $35 \%$ and about $10 \mathrm{~m}$ in radius. Four bound-state eigenfunctions were initialized from the theoretical dispersion relation in Figure 2: two eigenfunctions from the $n=1$ branch $\omega_{21} / \Omega=-0.191$ and $\omega_{51} / \Omega=-0.171$ and two eigenfunctions from the $n=2$ branch, $\omega_{22} / \Omega=-0.309$ and $\omega_{52} / \Omega=-0.266$. The wave energy was conserved to $0.0015 \%$ over $1024 \mathrm{LH}$ periods.

\subsection{Time-Frequency Analysis}

The evolution of the electrostatic potential $\varphi\left(k_{x}, k_{y}, t\right)$, for a perpendicular slab $(x, y)$ was stored over $512 \mathrm{LH}$ periods. The potential was sampled at a rate of 16 times per a $\mathrm{LH}$ period to allow spectral analysis of the dynamical evolution. The high sample rate was required to resolve high-frequency cavity eigenmodes which were a result of the deep (35\%) density depletion. The temporal history of the electrostatic potential was windowed using an Exact Blackmann-Harris window [Harris, 1978] to minimize spectral leakage. This window is continuous in function and first derivative at the boundary and falls off at $18 \mathrm{~dB}$ per octave which makes it ideal for investigating numerical simulations where the eigenfrequencies are discrete and the noise typically appears near roundoff. The temporal signal was Fouriertransformed $\varphi\left(k_{x}, k_{y}, t\right) \rightarrow \varphi\left(k_{x}, k_{y}, \omega\right)$ and the electric field power power spectral density was computed from

$$
P\left(k_{x}, k_{y}, \omega\right)=\left(k_{x}^{2}+k_{y}^{2}\right)\left|\varphi\left(k_{x}, k_{y}, \omega\right)\right|^{2} .
$$

The dispersion relation for the simulation is shown in Plate 1 for $k_{x}=0$. The dashed line at $\omega / \Omega \approx 0.5$ is the ambient LHR. The spectral representations were truncated outside the cylindrical region $k_{x}^{2}+k_{y}^{2}=$ $8 / 9\left(N_{x} / 2\right)^{2}$ to allow partial dealiasing of the nonlinear terms [Orszag, 1971]. The power converges to the $\omega_{\mathrm{LH}}$ as $k_{y} \rightarrow \infty$ indicating that the simulation correctly reproduces the resonance behavior of the theoretical model. Additionally, weaker images of the resonance structure appear at $\omega_{11} \pm \omega_{\text {LH }}$ produced by wave-wave scattering off the $m=1, n=1$ eigenfunction at $\omega_{11} / \Omega=2.93$. A distinctive feature of the simulation dispersion relation are the power bands where many Fourier basis vectors oscillate with the same frequency. This is the signature of a coherent stationary state which is composed of many Fourier basis vectors. A narrow band filter was applied to three of these power bands. The results were inverse transformed $\varphi\left(k_{x}, k_{y}, \omega\right) \rightarrow \varphi(x, y, t)$ to determine the spatiotemporal evolution of the power band. Two snapshots of the temporal evolution of $\varphi(x, y, t)$ for each power band is shown in Figure 4. Each column corresponds to a particular type of eigenmode and the rows correspond to the times $t / \tau_{\mathrm{LH}_{H}}=0$ and $t / \tau_{\mathrm{LH}}=0.13$ where $\pi_{\mathrm{LH}}=2 \pi / \omega_{\mathrm{LH}}$.

3.1.1. Bound state: $\omega / \omega_{\mathrm{LH}}=0.64$. This power band $(\omega / \Omega=0.32)$ occurred below the ambient LHR. The spatial structure shows an $m=2$ azimuthal dependence with $n=2$ nodes within the density depletion. The temporal evolution indicates that it rotates in a left-handed sense about the magnetic field. Additionally, the radial structure is well confined within the density depletion.

3.1.2. Propagating state: $\omega / \omega_{\mathrm{LH}}=2.26$. This power band $(\omega / \Omega=1.13)$ occurred above the ambient LHR. The spatial structure shows an $m=1$ azimuthal dependence with $n=2$ nodes within the density depletion. The temporal evolution indicates that it rotates in a right-handed sense about the magnetic field. Additionally, the radial structure leaks out of the density depletion. However, the radial wavelength of the mode is longer outside the depletion than inside. This indicates that the electric field in enhanced within the depletion.

3.1.3. Reflected state: $\omega / \omega_{\text {LH }}=1.58$. This power band $(\omega / \Omega=0.79)$ occurred above the ambient LHR. The spatial structure shows an $m=2$ azimuthal dependence outside the depletion. However, inside the depletion there is a $m=3$ azimuthal dependence with $n=2$ nodes within the density depletion. This suggests that the $m=2$ wave is evanescent within, or reflected from the density depletion. The temporal evolution indicates that external structure rotates in a left-handed sense about the geomagnetic field, while the internal structure rotates in a right-handed sense about the geomagnetic field. The azimuthal phase velocity of the internal structure is about two thirds that of the external structure.

\subsection{Discussion of the Simulation}

The qualitative results of the simulation agree with the linear eigenmode analysis. The eigenvalues and resonances of the linear analysis may be compared with the numerical simulation as a further consistency check. The frequency of the bound state $\omega / \Omega=0.32$ is consistent with the predicted $\omega_{22} / \Omega=-0.32$ for a $m=2$, $n=2$ left-handed bound state. The frequency of the propagating mode $\omega / \Omega=1.13$ is consistent with the predicted eigenvalue $\omega_{12} / \Omega=1.03$ for a $m=1, n=2$ right-handed resonance. The linear eigenmode anal- 


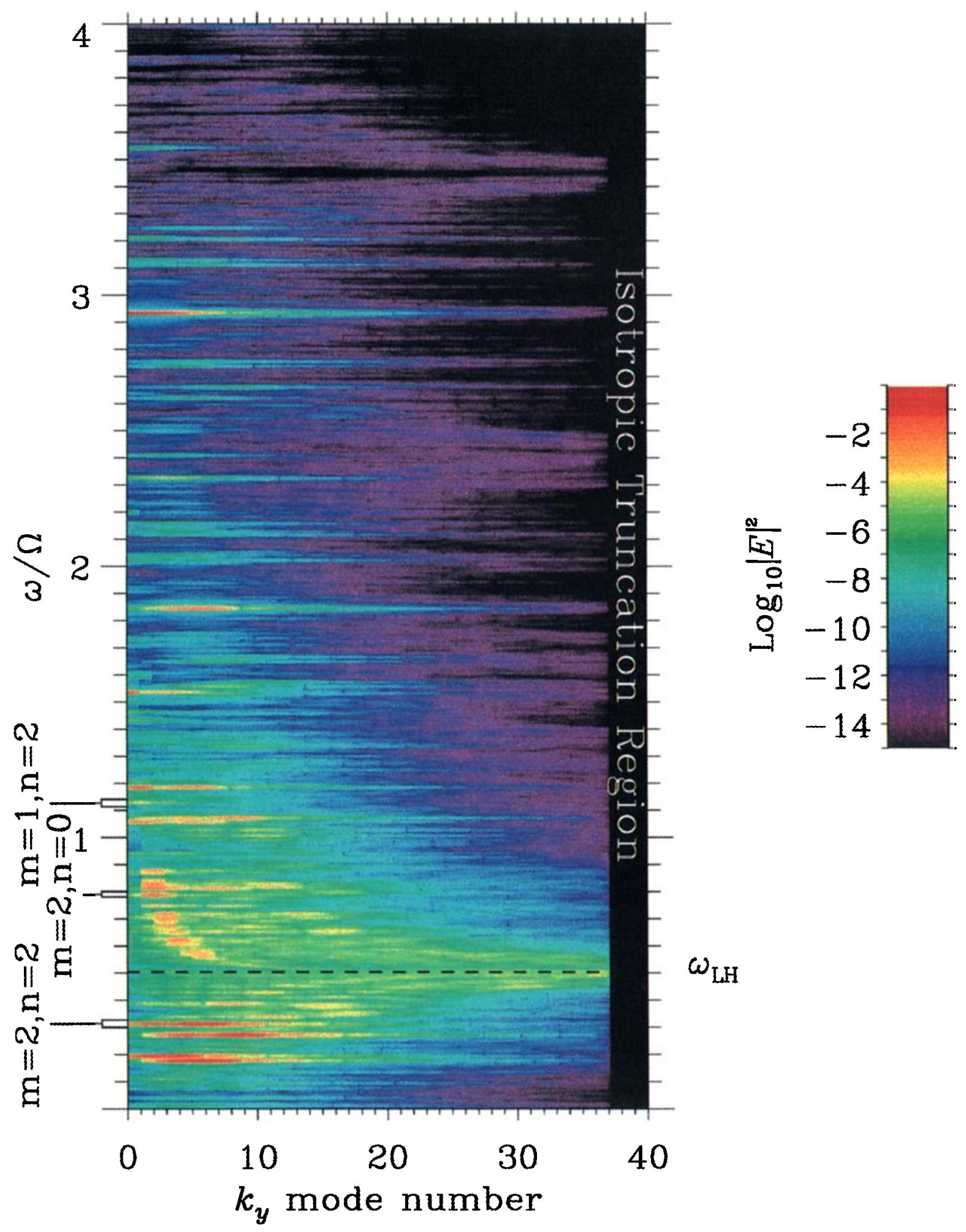

Plate 1. A logarithmic color scale representation of the power spectral density of $E^{2}$ in arbitrary units for $k_{x}=0$, illustrating the dispersive properties of a numerical simulation with $\Delta=-0.35$. The frequency is normalized to the magnetized LHR $(\Omega)$ and $k_{y}$ is in units of the fundamental wavenumber. The lower hybrid resonance is the dashed line at $\omega / \Omega \approx 0.5$. 

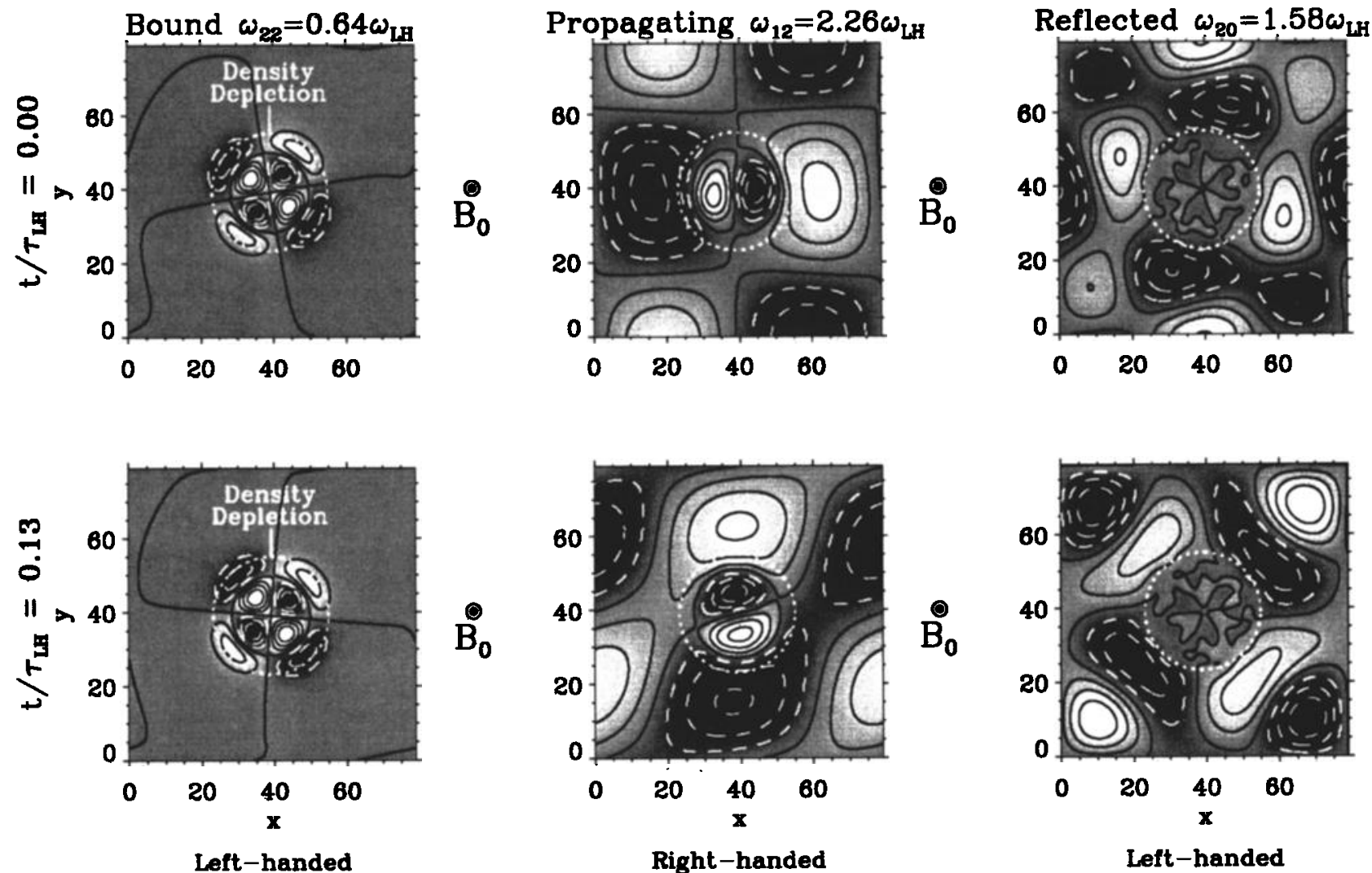

Figure 4. Two snapshots of temporal evolution of the power bands indicated in Plate 1. Each column corresponds to a particular type of eigenmode, and the rows correspond to the times $t / \tau_{\text {LH }}=0$ and $t / \tau_{\text {LH }}=0.13$

ysis and the full nonlinear simulations are consistent to within a few percent. The differences may be explained as follows: first a smoothed parabolic profile was used in the numerical simulation and second the linear eignvalue analysis was carried out for unbounded space whereas periodic boundary conditions are applied in the simulation.

The third power band discussed $(\omega / \Omega=0.79)$ is also consistent with the model. Consider that the left-hand rotating $m=2$ structure is excluded from within the density depletion. This is indicative of a reflected state. The internal structure is consistent with the predicted frequency $\omega_{32} / \Omega=0.81$ of an $m=3, n=3$ right-hand rotating resonance. Thus the filtering technique was not adequate to select a single eigenmode in this instance and the result was a superposition of an $m=2$ left-handed reflected state and an $m=3, n=3$ righthanded state.

\section{Observations of Rotating LHSS}

The local frequency-wavenumber spectrum was successful in revealing new details about the nature of LHSS observed by the AMICIST and TOPAZ III sounding rockets [Pinçon et al., 1997]. The analysis demonstrated that the phase velocity of $\mathrm{LH}$ waves along the interferometer axis reversed direction as the payload crossed the centerline of the event. Assuming that the LHSS structures are localized in the two dimensions perpendicular to the magnetic field, the reversal of the phase velocity indicated that $\mathrm{LH}$ waves rotated in a right-handed sense about the geomagnetic field within the LHSS events.

Unfortunately, the analysis of the AMICIST data was restricted to waves above the LHR. The power below the LHR was severely contaminated by plasma waves propagating radially outward from the payload (payload waves). These waves were produced by payload potential fluctuations and are clearly not geophysical in origin. Geophysical and payload waves are distinguished by phase shift. Plasma waves produced by payload potential fluctuations propagate radially outward from the payload and have a measured phase shift of $\pm \pi$ (by the convention used by Pinçon et al. [1997] and here) whereas geophysical waves have phase shifts anywhere between $-\pi$ and $\pi$. Additionally, when payload waves dominate the spectrum, the coherence of the Fourier cross spectrum is near unity.

The agreement between theoretical predictions and AMICIST observations for waves above the LHR suggested a reexamination of the TOPAZ III sounding rocket data. There were indications that the LHSS 
waves below the LHR observed on TOPAZ III were a mixture of payload and geophysical waves. The histogram method of Beall et al. [1982] provided a technique for differentiating between these wave modes. The local frequency-wavenumber spectrum revealed that along the interferometer axis, the phase velocity of the waves above the LHR was opposite to the phase velocity of the waves below the LHR. Assuming that the waves above the LHR rotate in a right-handed sense (consistent with AMICIST observations), the TOPAZ III observations indicated that LH waves below the LHR rotate in the left-handed sense.

These observations are in accord with the theory presented in section 2 and simulations presented in section 3. The detailed agreement between theory, experiment, and simulation for the AMICIST event discussed by Pinçon et al. [1997] suggested a thorough examination of a TOPAZ III event. This section reexamines event I, discussed by Vago et al. [1992]. First, the wavelet transform technique is used to examine the event. The local frequency-wavenumber spectrum is calculated and compared with one obtained from an interferometer "flown" through the simulation discussed in section 3. The combined results support the conclusion that LHSS are composed of rotating eigenmodes: right-handed above the LHR and left-handed below the LHR.

\subsection{TOPAZ III}

The TOPAZ III sounding rocket was launched northward out of Poker Flat, Alaska, at 0911:54 UT on February 12, 1991. The ground speed of the payload was about $1.1 \mathrm{~km} / \mathrm{s}$ at an apogee of $1068 \mathrm{~km}$. The experiment passed over several auroral arcs during the 18-min flight and encountered several hundred LHSS events. Since this analysis focuses on the wave data and density depletion, the discussion of apparatus will be limited to the interferometer and Langmuir probe. Detailed discussions of telemetry and particle detectors may be found in the works of Arnoldy et al. [1992], Kintner et al. [1992], and Vago et al. [1992].

The interferometer consisted of the two orthogonal double-probe Weitzmann boom assemblies as shown in Figure 5. The differences in the sphere potentials (VLF12, VLF34, VLF65, VLF78) were measured in the frequency range of $10 \mathrm{~Hz}$ to $20 \mathrm{kHz}$ to allow interferometric analysis of the plasma waves. The density measurements were made with a 2.5 -cm fixed-bias $(+5 \mathrm{~V})$ Langmuir probe mounted on the VLF56 mast. The payload spin period was about $0.1 \mathrm{~Hz}$ with the spin axis oriented perpendicular to both the geomagnetic field and the rocket velocity (cartwheel attitude) as shown in Figure 5. The crossed antenna lay in a plane containing the rocket velocity vector and the geomagnetic field. Therefore both parallel and perpendicular electric potentials were measured. The saturation level of the each channel of the interferometer was set

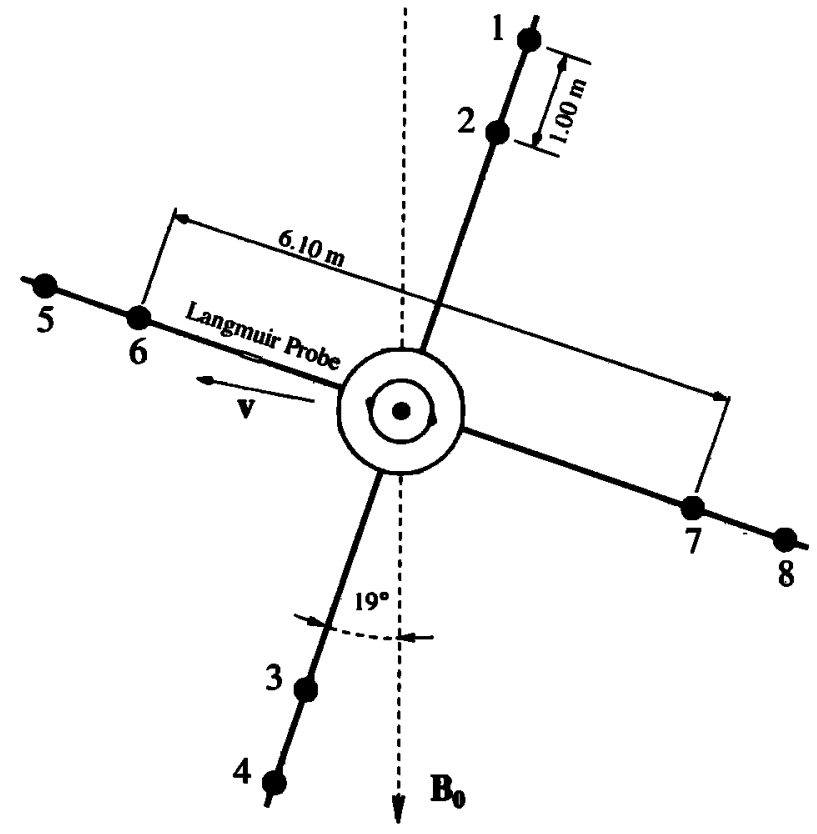

Figure 5. Schematic diagram (not to scale) of the TOPAZ III interferometer configuration and orientation at flight time $472.4 \mathrm{~s}$. The perspective is from fore to aft along the spin axis of the payload and the payload spins counterclockwise. The antenna lie in the plane containing the magnetic field $\mathbf{B}_{0}$, and payload velocity $\mathbf{v}$ (cartwheel attitude) which allows simultaneous measurements of parallel and perpendicular electric fields.

to $83 \mathrm{mV} / \mathrm{m}$ peak to peak. The aurora encountered was intense and saturated the potential measurements except when the booms were within $30 \mathrm{deg}$ of geomagnetic field. Therefore our discussion will be limited to the potentials across the mostly parallel booms.

\subsection{Local Frequency-Wavenumber Spectrum}

The LHSS are localized in space and their spatial size across the magnetic field is only a few or several tens of meters [Vago et al., 1992]. Consequently, the corresponding rocket data has transient features. The wavelet analysis is an appropriate technique for this situation.

To perform a wavelet transform, we first need to adopt a wavelet prototype function $g(t)$. Then, the wavelet transform of a signal $E(t)$ is defined by

$$
\begin{gathered}
E(a, \tau)=\frac{1}{\sqrt{a}} \int_{-\infty}^{\infty} d t E(t) g^{*}\left(\frac{t-\tau}{a}\right) \\
a>0,
\end{gathered}
$$

where $g^{*}$ is the complex conjugate of $g$. The variables $\tau$ and $a$ are the localization and dilatation parameters, respectively. The Morlet function [Goupillaud et al., 1984]

$$
g(t) \approx \pi^{-1 / 4} e^{-t^{2} / 2} e^{-i \omega_{0} t},
$$

where $\omega_{0}=5.36$ has been chosen for the time-frequency 
representation of the electric field. This function is a sinusoid of frequency modulated by a Gaussian envelope and has a simple relation between $a$, the dilatation parameter, and $f$, the usual frequency

$$
f=\frac{f_{0}}{a},
$$

where $f_{0}=\omega_{0} / 2 \pi$. It has been shown [Dudok de Witt et al., 1995; Pinçon et al., 1997] that the wavelet analysis may be combined with the local frequencywavenumber spectrum estimation technique developed by [Beall et al., 1982]. First, the wavelet cross-spectrum $C_{d}(f, \tau)$ is defined as follows:

$$
\begin{aligned}
C_{d}(f, \tau) & =E_{12}(f, \tau) E_{43}^{*}(f, \tau) \\
& =\left\|C_{d}(f, \tau)\right\| e^{i \vec{k}_{d}(f, \tau) \cdot \vec{d}},
\end{aligned}
$$

where $\vec{d}$ is the separation vector between the two double probes 1-2 and 3-4. Then the wavelet local frequency wavenumber is defined as

$$
P(f, k)=\left\langle C_{0}(f, \tau) \delta\left[k-k_{d}(f, \tau)\right]\right\rangle .
$$

The delimiters () denote an average over the time localization parameter $\tau$. There are three main advantages to replacing the Fourier transform by the wavelet transform. First, the wave field is described in terms of plane wave packets instead of plane waves and thus stationarity is no longer required. Second, the increased time resolution improves the statistical robustness of the spectral estimates. Third the resolution is optimal at each frequency.

\subsection{Event I}

Event I occurred at 472.4-s flight time within a region of intense electron precipitation. A sharp cutoff in the power spectra at $f=4.6 \mathrm{kHz}$ prior to event I provided an estimate of the ambient LHR. Additionally, this was consistent with $f_{\mathrm{LH}}=4.5 \mathrm{kHz}$, calculated using measured magnetic field $B_{0}=0.36 \mathrm{G}$, and the ion densities $n_{\mathrm{O}^{+}}=3.9 \times 10^{3} \mathrm{~cm}^{-3}, n_{\mathrm{H}^{+}}=350 \mathrm{~cm}^{-3}$, and $n_{\mathrm{He}^{+}}=60 \mathrm{~cm}^{-3}$ measured by the particle experiments [Vago et al., 1992].

The interferometer orientation during event $I$ is shown in Figure 5. The mostly perpendicular channels (VLF56 and VLF87) were saturated by the burst. Therefore the discussion will be limited to the mostly parallel antennas (the VLF12 and VLF34 channels) which were at an angle of $19^{\circ}$ with respect to the magnetic field. The signature of the LHSS is shown in Figure 6. Figure 6 shows the fixed biased $(+5 \mathrm{~V})$ Langmuir probe current which measured a $75 \%$ drop in current associated with the LHSS. The VLF12 and VLF43 channels measured two distinct changes in activity. First, there was a sudden increase in wave activity at $\mathbf{4 7 2 . 4 1}$ $s$ followed by a sudden decrease in activity at $\mathbf{4 7 2 . 4 5}$ s. The frequency response of the VLF12 and VLF43 channels below $1.25 \mathrm{kHz}$ is not geophysical and may
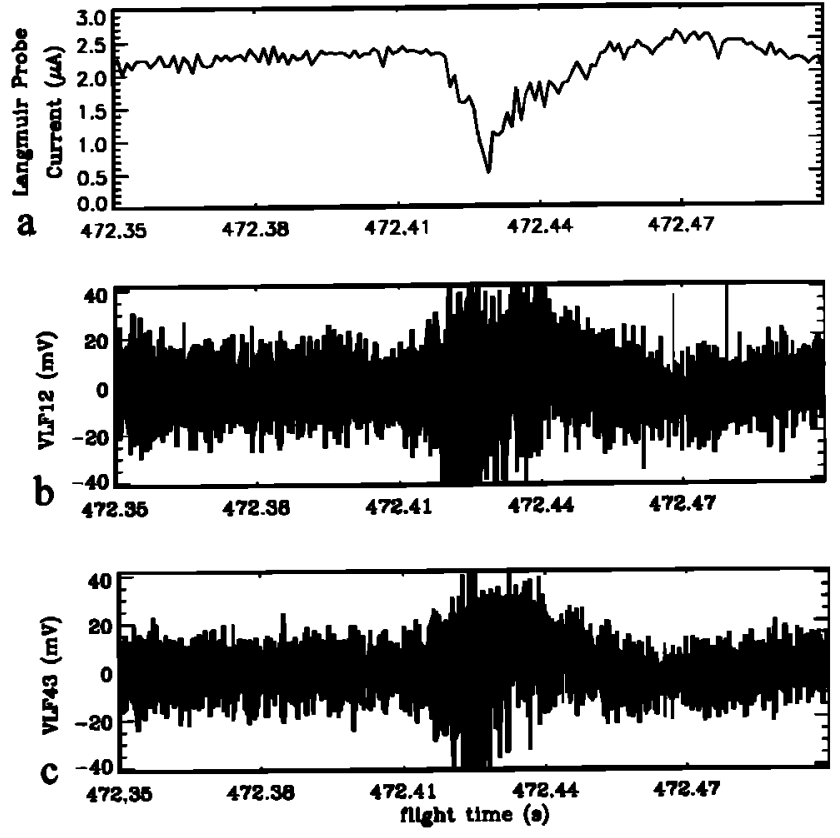

Figure 6. The time signature of the LHSS observed at $472.4 \mathrm{~s}$ flight time. (a) The fixed biased $(+5 \mathrm{~V})$ Langmuir probe current shows a $75 \%$ drop in measured current at this time indicating a deep density depletion. The LHSS structure is characterized by the sudden increase (472.41 s) and then decrease (472.45 s) in activity measured by the (b) VLF12 and (c) VLF43 channels.

be attributed to payload-plasma interactions [Neubert et al., 1986; Vago et al., 1992].

\subsection{Wavelet Analysis of Event I}

The wavelet local spectrum estimation technique was applied to the VLF12 and VLF43 channels measured by TOPAZ III during event I. The modulus of the wavelet cross spectrum of the mostly parallel channels VLF12 and VLF43 during event $I$ is shown in Plate 2. Power is in a logarithmic color scale representation, the frequency (ordinate) is on a logarithmic scale (base 2) and the flight time (abscissa) is on a linear scale. The frequency range is four octaves wide $(1.25-20 \mathrm{kHz})$. The maximum frequency is the Nyquist frequency, and the frequency range below $1.25 \mathrm{kHz}$ is of no geophysical interest since these waves may be attributed to payload emissions. The horizontal dashed line indicates the LHR estimated from the Fourier analysis of the VLF hiss prior to event I. The power is clearly attenuated below the LHR prior to and just after event I. During event I, from 472.15 to $472.45 \mathrm{~s}$ there is a strong intensification below the LHR.

The phase information of the wavelet cross spectrum may be used to compute the local frequency-wavenumber spectrum $P\left(f, K_{14}\right)$, where $K_{14}$ in $\mathrm{m}^{-1}$ is the projection of the wave vector onto the separation vector between the midpoints of the 1-2 and 3-4 double probes. 


$$
K_{14}=\frac{\vec{k} \cdot \overrightarrow{d_{14}}}{2 \pi\left\|\vec{d}_{14}\right\|} \quad\left\|\vec{d}_{14}\right\|=7.1 \mathrm{~m}
$$

Plate 3 (top) is logarithmic color scale representation of the local frequency-wavenumber spectrum for event I. Frequency is on a logarithmic scale (base 2) and wavenumber is in $\mathrm{m}^{-1}$ in the range $-1 /(2 d)$ to $1 /(2 d)$, which corresponds to a phase shift of $-\pi$ to $\pi$. The vertical dashed line separates positive phase velocities from negative phase velocities. The horizontal dashed line indicates the ambient LHR.

The data show a broad band intensification above the ambient LHR associated with primarily negative phase velocities. The wave numbers $K_{14}$ approach $-1 /(2 d)$ as the frequency approaches the LHR from above. Below the LHR there are four distinct intensifications associated with positive phase velocities: two near $2.5 \mathrm{kHz}$ at $K_{14}=0.025 \mathrm{~m}^{-1}$ and $K_{14}=0.042 \mathrm{~m}^{-1}$ and two near $1.8 \mathrm{kHz}$ at $K_{14}=0.02 \mathrm{~m}^{-1}$ and $K_{14}=0.04 \mathrm{~m}^{-1}$. The power near $K_{14}=0.06 \mathrm{~m}^{-1}$ is associated with broadband emissions from dc-1.25 kHz at a phase shift of about $\pi$. This phase shift indicates the waves are propagating outward from the payload and are not geophysical (not externally applied). The phase velocity changes discontinuously from negative values above the LHR to positive values below the LHR. This discontinuity has been observed previously by [Vago et al., 1992; Delory et al., 1997; Pinçon et al., 1997] in relation to LH phenomena and LHSS.

A double-probe interferometer was moved across the simulation discussed in section 3 at $1.1 \mathrm{~km} / \mathrm{s}$ based on the ground speed of the TOPAZ III rocket at apogee. The interferometer configuration was equivalent to the mostly parallel antenna of TOPAZ III during event I as shown Figure 5. Plate 3 shows a comparison between event I (top) and the interferometer data from the numerical simulation (bottom) of the logarithmic color scale representation of the local frequency-wavenumber spectrum below the ambient LHR. The frequency is on a logarithmic scale (base 2) and the wavenumber $K_{14}$, is on a linear scale. The horizontal dashed line indicates the ambient lower hybrid resonance calculated from the Fourier analysis of the VLF hiss prior to the event I.

The significant power in the simulation occurs below the LHR since only bound states were initialized. However, it has been demonstrated previously [Pinçon et al., 1997] that the waves above the LHR are consistent with simulations. The simulation interferometer data show left-hand rotating eigenmodes $(m, n)=(2,2)$ and $(m, n)=(5,2)$ near $2.5 \mathrm{kHz}$ and $K_{14}=0.025 \mathrm{~m}^{-1}$ and $K_{14}=0.04 \mathrm{~m}^{-1}$, respectively. Additionally, the lefthand rotating eigenmodes $(m, n)=(2,1)$ and $(m, n)=$ $(5,1)$ near $1.65 \mathrm{kHz}$ and $K_{14}=0.025 \mathrm{~m}^{-1}$ and $K_{14}=$ $0.04 \mathrm{~m}^{-1}$, respectively. These modes are associated with positive phase velocities. The local frequencywavenumber spectra of event I (top) and the simulation interferometer data (bottom) are in close qualitative agreement. Although the simulations are nonlinear, the power levels for the eignmodes are somewhat independent. This independence occurs because each individual eigenmode is an exact solution to the nonlinear system and the mode-mode coupling is weak for these amplitudes. However, the frequencies and wavenumbers of the eigenmodes are not independent. Once the parallel wavenumber $k_{z}$, the density depth $\Delta$, and the radial extent of the cavity $a$ have been chosen, the LHSS spectrum is fixed. Each eigenmode has a particular frequency and radial wavenumber. The discrete LHSS simulation spectrum has been qualitatively matched to four observational wave modes (consisting of four frequencies and four wavenumbers) with only three free parameters $\left(k_{z}, \Delta, a\right)$. The frequency and wavenumber of each individual eigenmode in the simulation spectrum cannot be adjusted independently to match the observational spectrum. The qualitative agreement between the observational and simulation spectra lends support to the interpretation that LHSS are composed of rotating eigenmodes.

\subsection{Discussion of the Observations}

The antenna for TOPAZ III were in a plane containing the magnetic field and the payload velocity. The component of phase velocity along the antenna did not change direction during the event. This observation is consistent with waves which rotate. Consider the component of phase velocity of a right-hand rotating eigenmode along the 1-4 antenna as the payload crosses the LHSS as diagramed in Figure 7. The phase velocity is from $4 \rightarrow 1$ (negative) from edge of the event to the centerline in the first half of the LHSS and remains from $4 \rightarrow 1$ (negative) from the centerline to the edge of

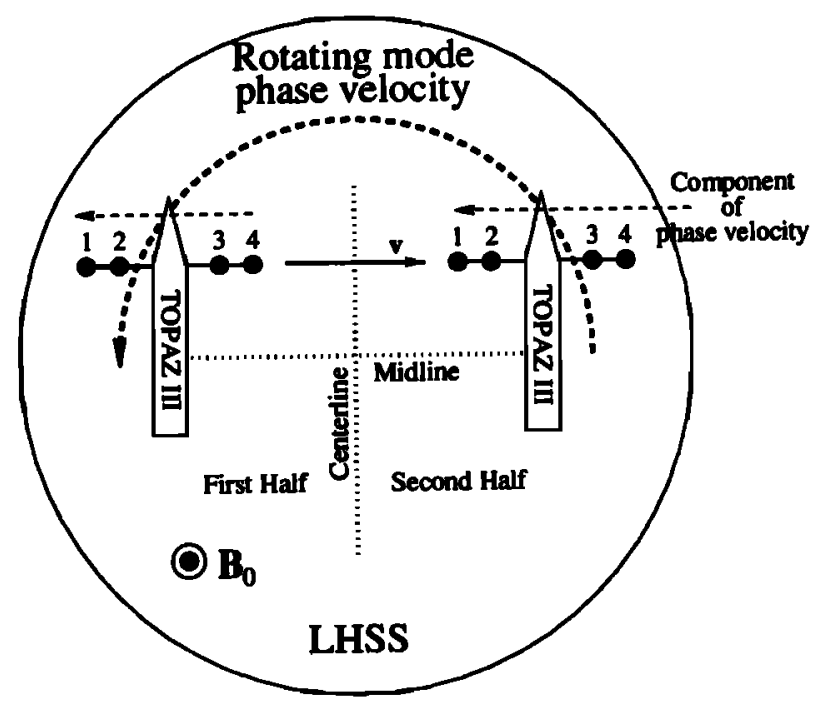

Figure 7. Schematic diagram of the measured component of phase velocity for a right-handed rotating mode. The 1-4 antenna measures the same direction of phase velocity for the wave in the first and second half of the event. Note that the 1-4 antenna is actually mostly parallel to the magnetic field $\mathbf{B}_{\mathbf{0}}$. 


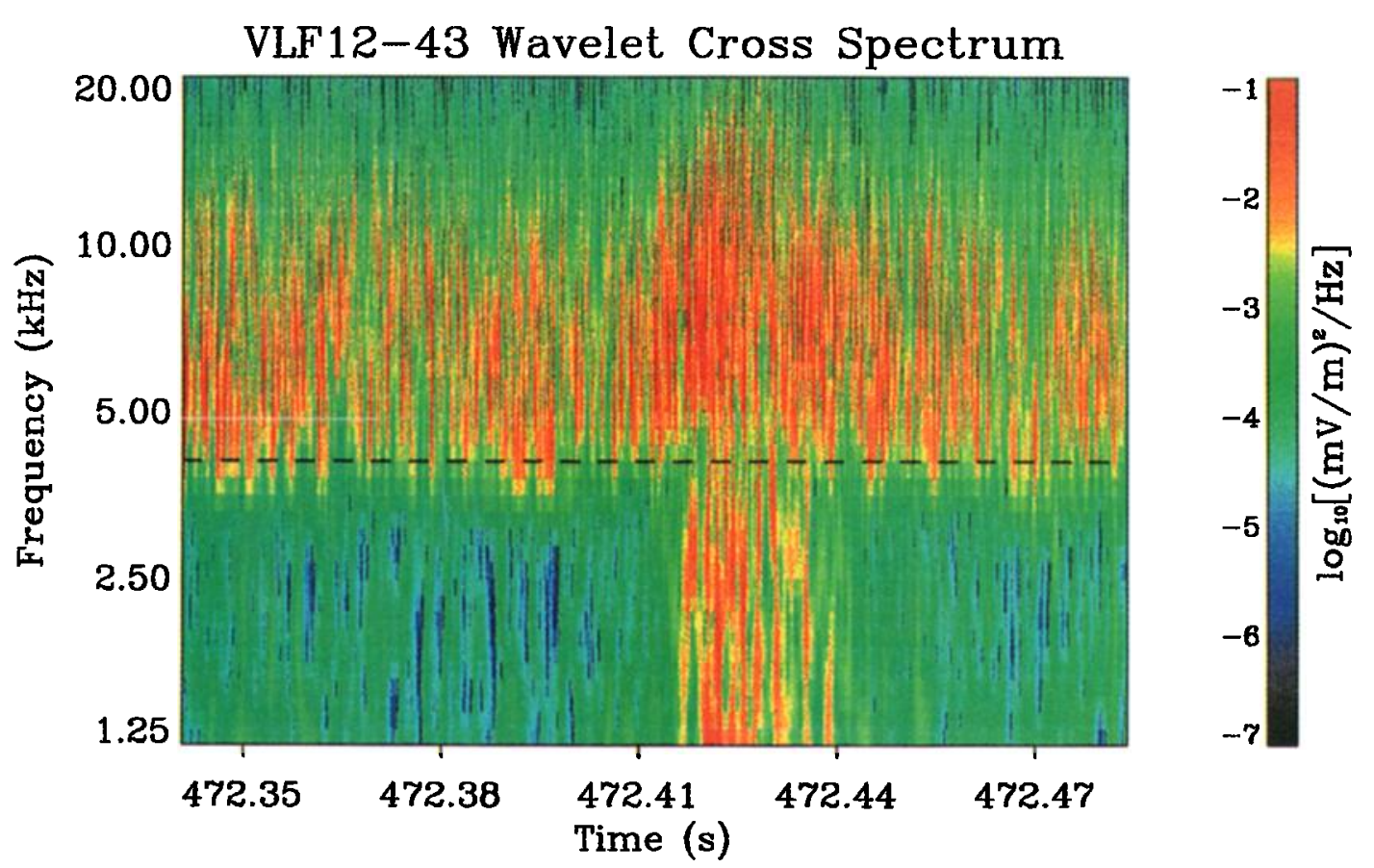

Plate 2. The logarithmic color scale representation of the modulus of the wavelet cross spectrum of the mostly parallel channels VLF12 and VLF43 during event I. The frequency is on a logarithmic scale (base 2), and the flight time is on a linear scale.

the event in the second half of the LHSS. Therefore it is clear that both the experimental interferometer data and the simulation interferometer data are consistent with rotating wave modes. However, the payload trajectory relative to the midline of the density depletion is unknown. If the rocket passed below the midline of the density depletion one would observe positive phase shift $1 \rightarrow 4$ for a right-hand rotating wave mode and a negative phase shift $4 \rightarrow 1$ for a left-hand rotating wave mode. Therefore the absolute sense of rotation of the waves about the magnetic field remains ambiguous. Several LHSS were examined with both orientations of phase velocity for the waves above the LHR. In each case the waves below the LHR had the opposite phase velocity to the waves above the LHR.

The fixed-biased Langmuir probe on the TOPAZ III sounding rocket responded dramatically to event I, registering a $75 \%$ drop in the probe current. For good agreement between the experiment and simulation interferometer wave spectra, a $30-40 \%$ parabolic density depletion was required in the numerical simulations. This appears to substantiate the large drop in Langmuir probe current observed on TOPAZ III. However, it must be emphasized that the Hall term depends explicitly on the density gradient not the absolute value of the density. Thus the numerical results are somewhat profile dependent. The discontinuity in phase velocity at the LHR is the signature of the Hall term mentioned in section 2 . The Hall frequency shift caused by the density gradient is dependent on the direction of propagation. For a cylindrical density depletion, right-hand rotating waves are shifted upward in frequency, whereas left-hand rotating waves are shifted downward in frequency.

It is apparent from the local frequency wavenumber spectrum that the traditional Fourier cross-spectral analysis will usually fail to illuminate the situation when applied to the LHSS data. Traditional Fourier crossspectral techniques are severely limited by the assumption of a single plane wave at each frequency. Clearly, this assumption is not correct for the bound state eigenfunctions shown in Figure 3. Furthermore, as shown in Figure 2 for a single parallel wavenumber, the density of eigenstates of the LHSS increases as the frequency approaches the LHR. Since the eigenstates near the LHR have the same frequency (energy) asymptotically, there is a vanishingly small probability of exciting a single eigenstate at the LHR. The assumption of a single plane wave at each frequency is simply not true as the frequency approaches the LHR. Thus the structure of the eigenstates should produce a distinct drop in the coherence at the ambient LHR when standard Fourier cross-spectral techniques are used [Vago et al., 1992; Delory et al., 1997]. Last the wavelet basis functions provide optimal spctral resolution at each frequency and eliminate the restriction of stationary plane waves inherent in traditional Fourier analysis. These properties give wavelet techniques at distinct advantage over Fourier techniques in the analysis of transient phenomena.

\section{Summary and Discussion}

Lower hybrid solitary structures have been observed on at least four sounding rockets MARIE [Labelle et al., 


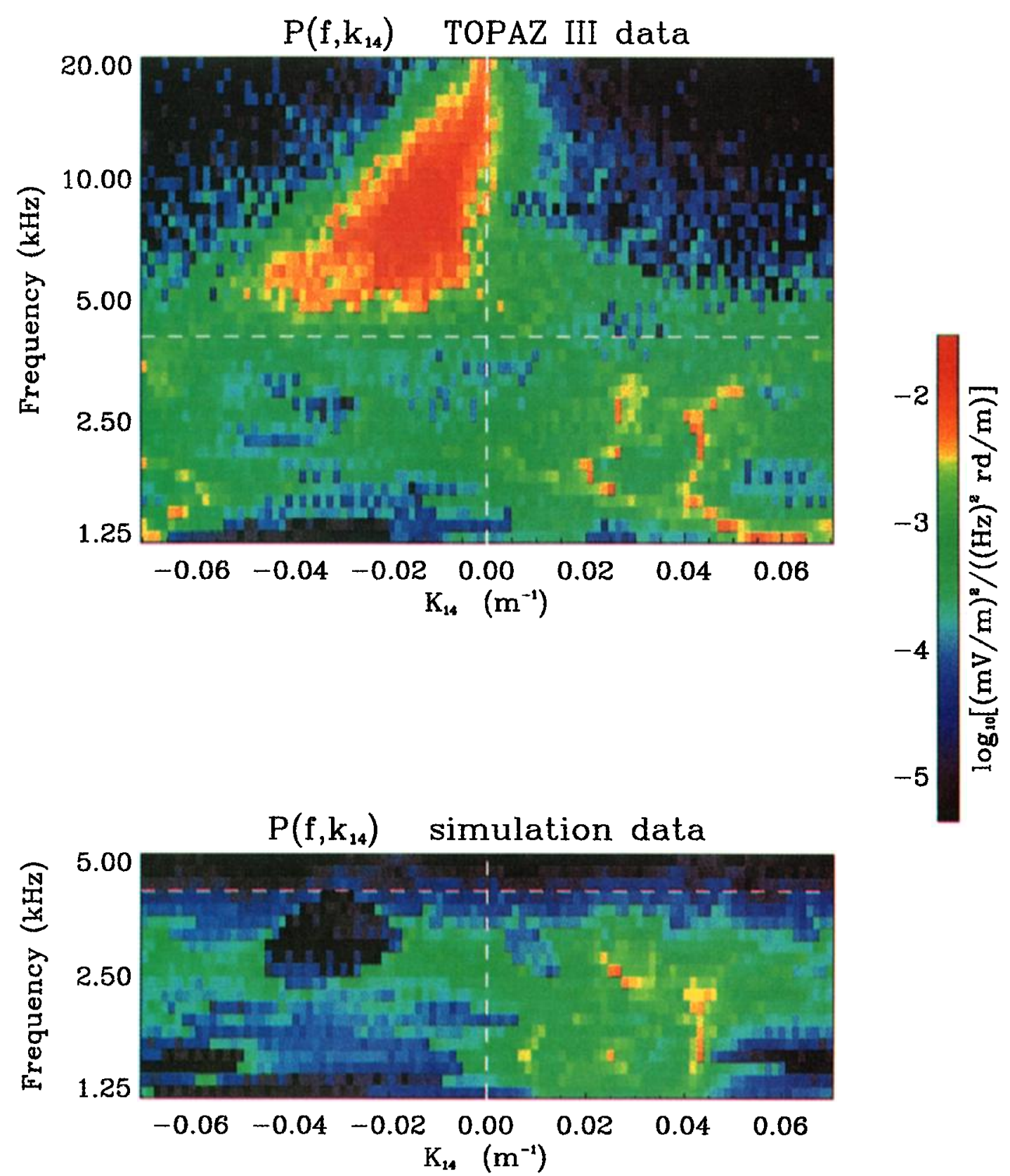

Plate 3. (top) Logarithmic color scale representation of the local frequency-wavenumber spectrum for event I. (bottom) Logarithmic color scale representation of local frequency-wavenumber spectrum below the ambient LHR for the interferometer data from the numerical simulation. The frequency is on a logarithmic scale (base 2) and the wavenumber $K_{14}$, is on a linear scale. The horizontal dashed line indicates the ambient lower hybrid resonance calculated from the Fourier analysis of the VLF hiss prior to the event I.

1986], TOPAZ III [Kintner et al., 1992; Vago et al., 1992], AMICIST [Pinçon et al., 1997], and PHAZE II (Bonnell et. al. submitted manuscript 1997) and two satellites Viking [Block et al., 1987; Pottelette et al., 1988, 1992] and Freja [Eriksson et al., 1994; Dovner et al., 1994]. This phenomenon appears to be a common feature of the nightside auroral zone.

The model discussed in this paper has been compared with LHSS observed by sounding rockets in cartwheel (TOPAZ III, herein) and propeller (AMICIST [Pinçon et al., 1997]) attitude. The detailed agreement between theory and observation suggest that the physics of LHSS is dominated by ail electron Hall current produced by $\mathbf{E} \times \mathbf{B}$ drifting electrons and unmagnetized ions. Solutions to the model were obtained by linearizing the dynamical system about a parabolic density depletion. Below the LHR the eigenfunctions are discrete and rotate in a left-handed sense about the magnetic field. Above the LHR the eigenfunctions are continuous and rotate in a right-handed sense about the magnetic field. 
The long-term (1000 lower hybrid periods) stability of the linear eigenfunctions in the nonlinear simulations suggest that the primary features of LHSS may be explained through the resonant scattering of $\mathrm{LH}$ waves off preexisting density depletions. Observations of LHSS from the TOPAZ III sounding rocket are consistent with rotating structures and are in agreement with interferometer data from the numerical solutions.

Lower hybrid collapse has been observed under restrictive conditions in various numerical simulations [Shapiro et al., 1993; Seyler, 1994; Shapiro et al., 1995]. Although the collapse of LH wave provides possible explanation for density depletions, the theories have two serious shortcomings: First, the timescale for collapse is $t_{c} \sim 1-10 \mathrm{~ms}$ [Shapiro et al., 1995], but the observed LHSS are coherent and quasi-stationary over at least 20-50 ms. Second, the balance between the ponderomotive force and the pressure cannot account for the observed depth of the density depletions [Robinson et al., 1996]. No lower hybrid collapse has been observed in simulations where initial conditions have been adjusted to reproduce observed parameters. The simulation power spectra shown in Plate 1 demonstrates that the wave-wave interaction between rotating eigenfunctions is weak. Additionally, it can be demonstrated that individually, the bound states are exact nonlinear eigenfunctions of a parabolic density profile. The eigenfunctions above the LHR are also exact nonlinear solutions, but individually they have unbounded energy and may only be considered within the context of a wave packet description. The collapse concept appears unnecessary for describing the electric fields associated with LHSS if the density depletions are produced by other mechanisms. Therefore we conclude that $\mathrm{LH}$ wave collapse does not occur for typical nightside auroral conditions, but the existence of $\mathrm{LH}$ collapse in nature is still an open question.

The model described herein has provided a description of the electric fields observed as LHSS. However, the acceleration mechanism associated with the ions has not been addressed. We suspect that the production of TAI is connected with the details of the rotating eigenfunctions. Although several mechanisms have been suggested for TAI, only quasilinear diffusion [ $A \boldsymbol{n}$ drulis et al., 1996] has been examined in the context of rotating electric field structures. A simple Landau resonance $\omega / m-l \Omega_{\mathrm{i}}$ between a lth harmonic of the ion cyclotron frequency and the azimuthal phase velocity of rotating eigenmode could accelerate the ion until its gyroradius extended outside the localized field structure. This mechanism suggests that ions would be most efficiently accelerated by the left-handed bound state eigenmodes. These waves are highly localized (trapped), coherent and rotate about the geomagnetic field in the same sense as the ions. Bulk ions in the auroral zone typically have energies on the order of $0.3 \mathrm{eV}$, corresponding to gyroradii $\rho_{\mathrm{O}^{+}} \approx 6 \mathrm{~m}$ and $\rho_{\mathrm{H}^{+}} \approx 1.6$ $\mathrm{m}$ for the primary ion species. Consider a LHSS with an effective radius of $20 \mathrm{~m}$. The gyroradius of the ion orbiting the center of the density depletion extends beyond the radius of the LHSS when $T_{\mathrm{O}^{+}} \approx 3 \mathrm{eV}$ and $T_{\mathrm{H}^{+}} \approx 50 \mathrm{eV}$. Off center ion orbits may extend outside the LHSS. The acceleration mechanism for this situation might be explained by transit time effects, where properly phased ions are incrementally accelerated each time the ion passes through the LHSS. Finally, a nonlinear Landau resonance between the ion cyclotron frequency and the beat wave of two rotating eigenmodes may also produce the observed TAI.

Traditional Fourier spectral techniques are limited by the assumption of a single stationary plane wave at each frequency. This assumption is clearly not appropriate for the transient phenomena of LHSS. The wavelet functions are clearly a more appropriate basis for the decomposition of transient phenomena. The histogram method [Beall et al., 1982] removes the constraint of a single plane wave at each frequency. The combination of the wavelet basis and the histogram method produces a wavelet local frequency-wavenumber spectrum $[D u$ dok de Witt et al., 1995; Pinçon et al., 1997] which is ideal for stituations involving transient phenomena with degenerate or near degenerate frequencies. We suspect that the local frequency-wavenumber spectrum analysis will elucidate other transient phenomena.

This work has provided new insight into the phenomena known as LHSS. However, we have no explanation for the origin of the density depletions. Additionally, a direct mechanism for the excitation of bound stated below the LHR does not exist within the context of the electrostatic model. These states are probably excited through resonant scattering of long wavelength magnetosonic modes from the density depletion. A more complete description of the scattering problem with a concentration on the continuum eigenfunctions will be presented in a future paper.

Acknowledgments. The authors would like to thank Jason Franz for useful discussions concerning interferometry. Useful discussions with Greg Delory, Kristina Lynch, and Steve Rosenberg are also gratefully acknowledged. This work of P. W. Schuck and C. E. Seyler was supported by NASA grant NAGW-4594. The work of J. W. Bonnell and P. M. Kintner was supported by NASA grants, NAG5-601, NAG5-691, and NAG5-5008.

The Editor thanks J. Huba and another referee for their assistance in evaluating this paper.

\section{References}

Abramowitz, M., and I. A. Stegun (Eds.), Handbook of Mathematical Functions, Appl. Math. Ser., Vol. 55, Nat. Bur. of Stand., Washington, D. C., 1964, [Reprinted with corrections by Dover, New York, 1972].

Andrulis, R. S., C. E. Seyler, and N. F. Otani, Growth of short wavelength lower hybrid modes in a density depletion and transverse acceleration of ionospheric ions, $J$. Geophys. Res., 101 (7), 15,723-15,735, 1996.

Arnoldy, R., K. Lynch, P. M. Kintner, J. Vago, S. Chesney, T. E. Moore, and C. J. Pollock, Bursts of transverse ion 
acceleration at rocket altitudes, Geophys. Res. Lett., 19, 413-416, 1992.

Beall, J. M., Y. C. Kim, and E. J. Powers, Estimation of wavenumber and frequency spectra using fixed probe pairs, J. Appl. Phys., 53, 3933-3940, 1982.

Block, L. P., C. G. Fälthammer, P. A. Lindqvist, G. Marklund, F. S. Mozer, A. Pedersen, T. A. Potemra, and L. J. Zanetti, Electric field measurements on Viking: First results, Geophys. Res. Lett., 14, 435-438, 1987.

Chang, T., Lower-hybrid collapse, caviton turbulence, and charged particle energization in the topside auroral ionosphere and magnetosphere, Phys. Fluids B, 5(7), 26462656, 1993.

Chang, T., and B. Coppi, Lower hybrid acceleration and ion evolution in the suprauroral region, Geophys. Res. Lett., 8(12), 1253-1256, 1981.

Delory, G. T., R. E. Ergun, E. M. Klementis, C. W. Carlson, and J. P. McFadden, Measurements of short wavelength VLF wave bursts in the auroral ionosphere: A case for electromagnetic mode conversion, Geophys. Res. Lett., $24(9), 1131-1134,1997$.

Dovner, P. O., A. I. Eriksson, R. Boström, and B. Holback, Freja multiprobe observations of electrostatic solitary structures, Geophys. Res. Lett., 21(27), 1827-1834, 1994.

Dudok de Witt, T., V. V. Krasnosel'skikh, S. D. Bale, M. W. Dunlop, H. Lühr, and S. J. Schwarz, Determination of dispersion relations of quasi-stationary plasma turbulence using dual satellite data, Geophys. Res. Lett., 22, 2653 $2656,1995$.

Ergun, R. E., E. M. Klementis, G. T. Delory, C. W. Carlson, and J. P. McFadden, Plasma density observations during large-amplitude lower hybrid emissions, Eos Trans. AGU, $\gamma \gamma(44), 1994$.

Ergun, R. E., E. M. Klemetis, G. T. Delory, J. P. McFadden, and C. W. Carlson, VLF wave localization in the lowaltitude auroral region, Geophy. Res. Lett., 22(16), 20992102, 1995.

Eriksson, A. I., B. Holback, P. O. Dovner, R. Boström, G. Holmgren, M. André, L. Eliasson, and P. M. Kintner, Freja observations of correlated small-scale density depletions and enhanced lower hybrid waves, Geophys. Res. Lett., 21(27), 1843-1846, 1994.

Forest, E., and R. D. Ruth, Fourth-order symplectic integration, Physica D, 43, 105-117, 1990.

Gordeev, A. V., and L. I. Rudakov, Stability of a Plasma Contained by a Strongly Non-uniform Magnetic Field, Nucl. Fuszon, 10, 319-324, 1970.

Goupillaud, P. A., A. Grossmann, and J. Morlet, Cycle-octave and related transforms in seismic signal analysis, Geoexploration, 23, 85-102, 1984.

Harris, F. J., On the use of windows for harmonic analysis with discrete fourier transforms, Proc. IEEE, 66(1), 51 83, 1978.

Horowitz, J. L., Conical distributions of low-energy ion fluxes at sychronous orbit, J. Geophys. Res., 85, 2057, 1980.

Kingsep, A. S., Y. V. Mokhov, and K. V. Chukbar, Nonlinear skin effect in plasmas, Fzz. Plazmy, 10, 854-859, 1984.

Kintner, P. M., J. Vago, S. Chesney, R. L. Arnoldy, K. A. Lynch, C. J. Pollock, and T. E. Moore, Localized lower hybrid acceleration of ionospheric plasma, Phys. Rev. Lett., 68(16), 2448-2451, 1992.

Klumpar, D. M., Transversely accelerated ions: An ionospheric source of hot magnetospheric ions, $J$. Geophys. Res., 84, 7201, 1979.

Labelle, J., P. M. Kintner, A. W. Yau, and B. A. Whalen, Large amplitude wave packets observed in the ionosphere in association with transverse ion acceleration, $J$. Geophys. Res., 91, 7113, 1986.

Longcope, D. W., Theoretical studies of magnetohydrodynamic equilibrium and dynamics of a solar coronal loop, Ph.D. thesis, Cornell Univ., Ithaca, N. Y., 1993.

Lynch, K. A., R. L. Arnoldy, P. M. Kintner, and J. Bonnell, The AMICIST auroral sounding rocket - a comparison of transverse ion acceleration mechanisms., Geophys. Res. Lett., 23(23), 3293-3296, 1996.

Maggs, J. E., Coherent generation of VLF hiss, J. Geophys. Res., 81(10), 1707-1724, 1976.

Musher, S. L., and B. I. Sturman, Collapse of plasma waves near the lower hybrid resonance, Pis'ma Zh. Eksp. Teor. Fiz., 22(11), 537-542, 1975, [JETP lett. 22, 265-267, 1975.].

Neubert, T., G. Holmgren, E. Ungstrup, and K. Melgård, Waves with harmonic structure above and below the lower hybrid resonance observed on the centaur 35.001 and 35.002 rockets, Can. J. Phys., 64, 1437-1445, 1986.

Orszag, S. A., Numerical simulation of incompressible flows within simple boundaries, I, Galerkin (spectral) representations, Stud. Appl. Math., 50(4), 293-327, 1971.

Pécseli, H. L., K. Iranpour, O. Holter, B. Lybekk, J. Holtet, J. Trulsen, A. Eriksson, and B. Holback, Lower-hybrid wave cavities detected by the Freja-satellite, J. Geophys. Res., 101 (8), 5299, 1996.

Pinçon, J.-L., P. M. Kintner, P. W. Schuck, and C. E. Seyler, Observation and analysis of lower hybrid solitary structures as rotating eigenmodes, J. Geophys. Res., 102(8), 17283-17296, 1997.

Pottelette, R., M. Malingre, A. Bahnsen, L. Eliasson, K. Stasiewicz, R. E. Erlandson, and G. E. Marklund, Viking observations of bursts of intense broadband noise in the source regions of auroral kilometric radiation, $A n n$. Geophys., 6(5), 573-586, 1988.

Pottelette, R., R. A. Treumann, and N. Dubouloz, Generation of auroral kilometric radiation in upper hybrid wave-lower hybrid soliton interaction, J. Geophys. Res., 97(A8), 12,029-12,044, 1992.

Retterer, J. M., T. Chang, and J. R. Jasperse, Ion acceleration by lower hybrid waves in the suprauroral region, $J$. Geophys. Res., 91(A2), 1609-1618, 1986.

Robinson, P. A., A. Melatos, and W. Rozmus, Is there lower hybrid collapse at auroral latitudes?: Theory versus observations, J. Geophys. Res., 101(A10), 21,545-21,552, 1996.

Ronnmark, K., WHAMP-waves in homogenous, anisotropic, multicomponent plasmas, Tech. Rep. 179, Kiruna Geophys. Inst., Kiruna, Sweden, 1982.

Rudakov, L. I., C. E. Seyler, and R. N. Sudan, Electron and hall magnetohydrodynamics and magnetic field penetration in a plasma, Comm. Plasma Phys. Controlled Fusion, $14,171,1991$

Seyler, C. E., A mathematical model of the structure and evolution of small-scale discrete auroral arcs, $J$. Geophys. Res., 95(A10), 17,199-17,215, 1990.

Seyler, C. E., Lower hybrid wave phenomena associated with density depletions, J. Geophys. Res., 99, 19,513-19,525, 1994.

Shapiro, V. D., V. I. Shevchenko, G. I. Solov'ev, V. P. Kalinin, R. Bingham, R. Z. Sagdeev, M. AshoraAbdalla, J. Dawson, and J. J. Su, Wave collapse at the lower hybrid resonance, Phys. Fluids B, 5(9), 3148-3162, 1993.

Shapiro, V. D., G. I. Soloviev, J. M. Dawson, and R. Bingham, Lower hybrid dissipative cavitons and ion heating in the auroral ionosphere, Phys. Plasmas, 2(2), 516-526, 1995.

Sharp, R. D., R. G. Johnson, and E. G. Shelly, Observation of an ionospheric acceleration mechanism producing 
energetic (kev) ions primarily normal to the geomagnetic field, J. Geophys. Res., 82(22), 3324-3328, 1977.

Shelley, E. G., R. D. Sharp, and R. G. Johnson, Satellite observations of an ionospheric acceleration mechanism, Geophys. Res. Lett., 3, 654-657, 1976.

Sotnikov, V. I., V. D. Shapiro, and V. I. Shevchenko, Macroscopic consequences of collapse at the lower hybrid resonance, Fiz. Plazmy, 4(2), 253-257, 1978, [Sov. J. Plasma Phys. 4, 450-459 1978.].

Tam, S. W. Y., and T. Chang, The limitation and applicability of the Musher-Sturman equation to two-dimensional lower hybrid wave collapse, Geophys. Res. Lett., 22, 11251128, 1995.

Temperton, C., A generalized prime factor fft algorithm for any $n=2^{p} 3^{q} 5^{r}$, SIAM J. Sci. Stat. Comput., 13(3), 676686, 1992.

Vago, J. L., P. M. Kintner, S. W. Chesney, R. L. Arnoldy, K. A. Lynch, T. E. Moore, and C. J. Pollock, Transverse ion acceleration by localized lower hybrid waves in the topside auroral ionosphere, J. Geophys. Res., 97(A11), 16935-16957, 1992.

Zakharov, V. E., Collapse of Langmuir waves, $Z h$. Ekso. Teor. Fiz., 62, 1945, 1972, [Sov. Phys. JETP. 35, 908 1972.].

J. W. Bonnell, P. M. Kintner, and C. E. Seyler, School of Electrical Engineering, Cornell University, Ithaca, NY, 14853. (e-mail: johnb@ee.comell.edu; paul@ee.cornell.edu; seyler@ee.cornell.edu)

J.-L. Pinçon, 3A Avenue de la Recherche Scientifique, 45071 Orleans Cedex 2, France. (e-mail: jlpincon@cnrsorleans.fr)

P. W. Schuck, Laboratory of Plasma Studies, Cornell University, Ithaca, NY 14853. (e-mail: schuck@lps.cornell.edu)

(Received August 4, 1997; revised October 9, 1997; accepted October 10, 1997.) 